\title{
Review Paper: \\ The Prevalence of Fall and Related Factors in Iranian Elderly: A Systematic Review
}

\author{
Ali Akbar Pahlevanian¹ ${ }^{1}$, Reyhaneh Najarian², Sadegh Adabi³, *Mina Sadat Mirshoja ${ }^{1}$ [C]
}

1. Neuromuscular Rehabilitation Research Center, School of Rehabilitation, Semnan University of Medical Sciences, Semnan, Iran.

2. Student Research Committee, Department of Paramedical, School of Paramedical Sciences, Semnan University of Medical Sciences, Semnan, Iran

3. Department of Occupational Therapy, School of Rehabilitation, Semnan University of Medical Sciences, Semnan, Iran.

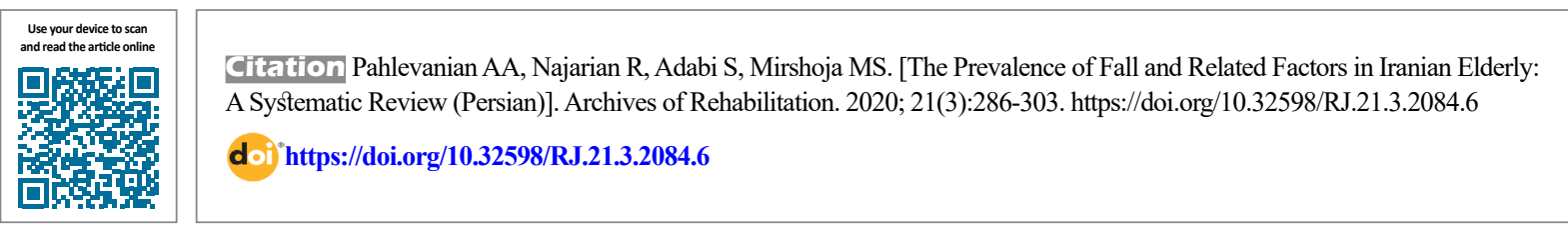

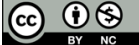

Received: 26 Dec 2019

Accepted: 11 Mar 2020

Available Online: 01 Oct 2020

Keywords:

Prevalence, Falling,

Elderly, Iran

\begin{abstract}
Objective Aging is one of the critical and growing stages in Iran. Falling is one of the most common problems in this period that leads to mobility, social limitations and even death. To increase the awareness and prevent secondary hazards due to falls, this study was conducted as a review article aiming the prevalence of falls based on evidence and related factors in Iranian elderly.

Materials \& Methods In this study, the Prism method includes research published from 2008 to 2018 focusing on the prevalence of falls based on evidence and related factors in the Iranian elderly and the keywords prevalence, falls, falls-related factors, the elderly In the scientific database of PubMed and Scopus, Google Scholar, SID, Magiran, Irandoc. After reviewing the studies, 394 articles were obtained and finally, by reviewing the abstracts, 14 articles were selected.

Results The results of Iranian studies were reported very differently. There was a significant relationship between aging, marriage, education, neurological diseases, painkillers, and home safety.

Conclusion The findings of this review study show an increase in the frequency of falls in the Iranian elderly that it is worthwhile to consider one of the health policies in this period of life to prevent and reduce the causes of falls at this age to reduce costs. Reduce the health caused by fall related injuries. Since prevention and education programs are not implemented in the elderly, the results of this study can help identify the importance of treatment costs and the harmful status.
\end{abstract}

\section{Extended Abstract}

\section{Introduction}

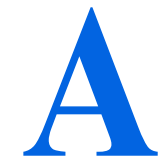

ging is one of the most critical stages in the lifespan of any person, which is associated with changes in physical, cognitive, social, and behavioral functions. More than $70 \%$ of the elderly population lives in developing countries. This rate is increasing with the improvement of health, the advancement of medical science, and the increase in life expectancy. According to the General Census of Population and Housing in Iran, about $2.8 \%$ of the population (6159676 people) in 2011 was older people. This rate increased to about $9.28 \%$ (7419091 people) in 2016, which is supposed to grow in the next 25 years.

Falls are the second leading cause of unintentional injury deaths worldwide. Every year, 424000 people worldwide die due to falls, which has been $4.6 \%$ in Iran. Falls do not

\section{* Corresponding Author:}

Address: Neuromuscular Rehabilitation Research Center, School of Rehabilitation, Semnan University of Medical Sciences, Semnan, Iran. Tel: +98 (23) 33654180

E-Mail: msj5831@yahoo.com 
usually cause serious injuries, but they can have serious physical and psychological consequences for the elderly. The most common are pelvic fractures, pain, immobility, fear of falling again, depression, and anxiety. These complications can negatively affect the quality of life of these people. Fear of falling syndrome is a common problem with a prevalence of $21 \%-85 \%$ among the elderly who perceive that a fall will occur. In a study on fear of falling, Deshpande et al. [13] found that about $25 \%$ of older people had no activity restriction, $59.6 \%$ had moderate restriction (functional restriction or avoidance in two activities or less), and $14.9 \%$ severe limitation (functional restriction or avoidance in three activities or more).

The World Health Organization has stated that cross-sectional data at any time cannot identify some of the conditions associated with fall injuries in developing and developed countries. This study investigates the prevalence of falls and related risk factors among the Iranian elderly by systematically reviewing local and national studies in recent years. This study seems to be one of the first studies in Iran in this field that can help health professionals describe, prevent, and plan effective treatment strategies to reduce and prevent falls. Hence, the research questions are as follows: What is the prevalence of falls in the Iranian elderly? What are the factors associated with falls in the Iranian elderly? Can demographic characteristics (e.g. age, gender, education, place of residence, related illnesses) increase the risk of falling? How do physical and psychological problems affect the risk of falling? What types of home environments increase the risk of falling?

\section{Materials and Methods}

In this study, the Prisma method was used to report systematic reviews of the studies. The search was conducted on articles published from 2000 to 2018 on the prevalence of falls and their risk factors among the Iranian elderly in PubMed, Scopus, Google Scholar, SID, and Magiran databases using the following keywords of "Prevalence", "Epidemiology", "Fall", "Slip", "Factors Related to Falls", "Accidental Falls", "Aged", "Elderly", "Older Elderly", "Older Adults", and "Iran". The articles in Persian or English conducted in Iran with descriptive-analytical design and available fulltext were included in the review. The incomplete articles with abstracts, dissertations, and posters were excluded. The Initial search yielded 172 articles, which reached 137 after considering the inclusion criteria. Then, by studying their abstracts, 23 articles that were in line with the objectives of the research were finally selected and reviewed.

\section{Results}

The prevalence rates of falls were $30.9 \%$ in Bojnourd City, 24.8\% in Khorramabad City, 35.1\% in Babol City, 82.31\% in Choram County, and $31.9 \%$ in Kashan City, during a 4-year retrospective study in Iran. Taheri Tanjani et al. [9] reported a prevalence of $25.5 \%$ in the elderly. Na'emani et al. [33] estimated the frequency of falls among the elderly living in Tehran as $66 \%$ once, $20 \%$ twice, $7 \%$ three times, $4 \%$ four times, $2 \%$ five times, and $1 \%$ six times. Mortazavi et al. [31] estimated the Mean \pm SD fear score of falling to be $11.3 \pm 11.07$ and reported a significant relationship between fear of falling and a history of falling $(\mathrm{P}<0.001)$. Because of the increasing population of the elderly in recent years, the fear of falling in the elderly has been considered an important issue. The basis for the prevention of falls is to avoid and reduce the risks associated with it. According to Taheri Tanjani et al. [9], the fall-related fracture rate was about $21.36 \%$, which was $5 \%-17 \%$ higher than in other countries. Davoodi et al. [26] also estimated the prevalence of falls in the elderly in Iran to be increased.

Marashi et al. [2] reported that the fall rate was $66.7 \%$ in the elderly aged $\geq 80$ years, $32.7 \%$ in the age group of $70-79$ years, and $14.9 \%$ in the age group of $<70$ years. The occurrence of falls was significantly associated with age. In Safavi Bayat et al.'s study, the risk of falling in the elderly was also increased with age, and there was a significant relationship between age and fall. The prevalence of falls was $30.3 \%$ in women and $13.9 \%$ in men. Jafarian Amiri et al. [20] stated that the risk of falls in the elderly aged $\geq 75$ years was 2.5 times higher than that in the elderly aged $<75$ years.

Ghanbari et al. reported that the fall rate was significantly related to the female gender and the prevalence of falls was $45.6 \%$ in women and $25.2 \%$ in men. Jafarian Amiri et al. however, found no significant difference in the prevalence of falls between men and women, which is consistent with the results of Corsinowi but against the results of Coimbra [20]. Nabavi et al. [11] found no significant relationship between gender and falling $(\mathrm{P}=0.28)$ like the results of Davoodi et al. and Taheri Tanjani and associates. Still, they reported that the rate of falls was higher in older people. Salarvand et al. [17] also reported a higher prevalence in women $(26.7 \%)$ than in men $(23 \%)$, where osteoporosis was the most common cause of falls in women. In another study, they estimated the prevalence of falls in older women at $51.5 \%$ and in men at $48.5 \%$.

Salavati et al. [35] and Nabavi et al. [11] did not find a significant relationship between education level and the prevalence of falling. In contrast, Davoodi et al. [26] found that educational level was significantly correlated to fall- 
ing, which may be due to the greater awareness of the risk factors for falling in the elderly with university education. Marashi et al. [2] and Abbasi et al. [29] showed that falls in less educated and uneducated older people were higher. Sayyah et al. [22] stated that the rate of falling in the group with primary education was higher (42.8\%).

Marashi et al. [2] found a significant relationship between marital status and falling in the elderly, where the highest rate of fall in the elderly without a spouse was higher (35.2\%). Salarvand et al. [17] reported that the rate of falls in married older adults $(20.2 \%)$ was less than the elderly without a spouse (34.6\%), and the fall rate was significantly associated with marriage. Davoodi et al. [26] showed that depressed older people were 8.2 times more likely to fall than healthy older adults. They also found that the incidence of falls in the elderly with cognitive impairment was 6.79 times higher than that in the elderly without cognitive impairment. The reason was considered to be the mental and functional limitations in performing daily activities. Shirini et al. [30] showed a significant relationship between the fear of falling with balance and anxiety in the elderly. According to them, psychological counseling for the elderly and helping them reduce their fear and manage their anxiety has a significant effect on increasing their balance.

The probability of falling in the elderly with urinary incontinence was $33.1 \%$ and with visual impairment $34.3 \%$. Marashi et al. [2] found no association between falling and having stroke and heart attack, but there was a significant relationship between sleeplessness and falls. Nabavi et al. [11] found a significant relationship between the risk of falls and low back pain $(\mathrm{P}=0.008)$, osteoarthritis $(\mathrm{P}=0.03)$, and hearing problems $(\mathrm{P}=0.02)$. Ghasemi et al. found the significant association of falling with orthostatic hypotension $(\mathrm{P}=0.002)$, dizziness $(\mathrm{P}=0.05)$, fecal incontinence $(\mathrm{P}<0.001)$, hearing impairment $(\mathrm{P}<0.001)$, visual impairment $(\mathrm{P}<0.001)$, lower extremity pain $(\mathrm{P}=0.027)$, and use of anticoagulants $(\mathrm{P}=0.017)$. The number of falls was strongly associated with poor eyesight, multiple medication use, chronic illness, the use of walking aids, dizziness, and impaired balance. A meta-analysis of 74 articles showed that risk factors for falls with a multifactorial etiology include the number of falls $(\mathrm{OR}=2.8)$, walking problems $(\mathrm{OR}=8.2,2.1)$, use of walking aids $(\mathrm{OR}=2.2,3.1)$, dizziness $(\mathrm{OR}=1.8,2.3)$; Parkinson disease $(\mathrm{OR}=2.7,2.8)$, and use of antiepileptic drugs $(\mathrm{OR}=1.9,2.7)$. In the study by Jafarian Amiri et al. [20], the incidence of falls in the elderly with a history of chronic disease was 4.5 times higher than that in the elderly without the disease. Hence, diseases such as heart disease, high blood pressure, hypotension, diabetes, seizures, headaches and dizziness, bone and joint diseases, impaired balance and gait, and vision problems in the el- derly can lead to disability in the elderly result in the falls accident. Salarvand et al. [18] also found a significant relationship between falls with visual impairment, heart attack, and osteoarthritis, but there was no link between diabetes and the risk of falling. Nabavi et al. [11] also found a significant relationship between the risk of falling and walking $(\mathrm{P}<0.008)$, sitting, and standing problems $(\mathrm{P}=0.009)$.

\section{Discussion and Conclusion}

One of the policies of the World Health Organization is to prevent falls and related injuries. According to this organization's report, the burden of unintentional injuries in developing countries is higher than that in developed countries, and falling is one of its most common causes. A review of past studies showed that the fear of falling could be as debilitating as falling or even worse. Many studies have shown a relationship between fear of falling and low functional mobility. Falling reduces people's sense of self-efficacy, and, as a result, they find themselves incapable of performing simple daily activities and walk slowly. It always reduces their sense of self-satisfaction and quality of life.

According to this study, the influential factors in falling can be divided into two categories of internal and external factors. The internal factors are age, gender, and associated diseases, while the external factors are the physical environment and the location of objects. Evidence of falls in older adults is varied. In this review study, three reviewed articles had estimated the prevalence of falling in the elderly in Iran compared to other countries. One study in 5 Iran cities showed that the prevalence of falls in the elderly in Iran was at a moderate rate compared to other countries. The prevalence of falls in other countries was reported to be between $11 \%$ and $42 \%$. Two other studies found that the rate of falls in the elderly in Iran was lower than the reported global rate. Considering the accuracy of recorded data, factors such as race and relatively low physical activity of the elderly in Iran can justify this difference, given that the number of fallrelated fractures is lower in Iran than in Western countries. Differences in study methods and periods, or the definition of a fall can also be factors related to the differences in the prevalence of falls between Iran and other countries.

Age has also been a common risk factor. Eight studies found the rate of falls to be significantly related to age. Five studies found a significant relationship between marital status and risk of falling. Four studies reported a higher prevalence of falls in women than in men, and 5 studies showed no difference. The discrepancy in results may be due to the difference in bone density between men and women. In three studies, there was no significant difference between educational level and falling, while 4 other studies reported 
a significant difference. Perhaps it can be attributed to the common problems of the elderly that all individuals and families are familiar with them. Injuries from falls not only negatively affect the performance, independence, and quality of life of the elderly but are also important predictors of the need for home care services and can lead to fear of falling, depression, anxiety, and social isolation in the elderly. Three studies reported a significant inverse correlation between physical activity and the fear of falling.

On the other hand, these studies showed that fear had a negative and significant relationship with daily activities. Inactivity and functional dependence as psychological variables are related to the reduced physical activity in the elderly. However, positive changes in the amount of physical activity can reduce the risk of chronic diseases and increase the quality of life of people. It also increases the efficiency and independence of the elderly and helps them control the various complications of aging and follow up with the treatments.

Nine studies showed that stroke and visual impairment were the most common risk factors, such that, during a follow-up of at least two years, the risk of falling in the elderly who had a stroke was 6 times higher, and in those with visual impairment, it was 5 times higher than that in controls. Because of the decrease in muscle movement (paresis) in people surviving stroke, the balance capacity in this group of patients decreases. Visual impairment, especially impaired proprioception and disability to differentiate between objects, causes the elderly to move inappropriately to the environment. This condition increases the likelihood of encountering obstacles and can be a significant risk factor for falling in the elderly. One study found no link between diabetes and falling. The low balance was another risk factor for falling. Five studies showed that psychological factors such as depression, dementia, guilt, and sadness due to urinary incontinence could play a role in falling. Living apart from other family members in nursing homes and limited access to the community affect psychological factors whose effects have not been adequately estimated. Depression increases the risk of falling. In general, the risk of depression is higher in the illiterate elderly and in the elderly who have lost their spouse for any reason. These seniors usually experience more socioeconomic status problems than seniors with an academic degree. As a result, this factor can increase their gradual isolation, loss of independence, loneliness, and mental distress. Poor sleep was another risk factor for falling. Injuries from falls are always more severe in the elderly than in the young. Therefore, they require more medical care and a longer rehabilitation period. Four studies showed that falls are associated with the type of used medication, especially antidepressants, fol- lowed by benzodiazepines, probably because the majority of the participants in these studies used short-acting benzodiazepines. Simultaneously, it seems that long-acting benzodiazepines are associated with more falls due to causing dizziness and decreased attention to the environment.

\section{Ethical Considerations}

Compliance with ethical guidelines

All ethical principles are considered in this article.

Funding

This research did not receive any grant from funding agencies in the public, commercial, or non-profit sectors.

Authors' contributions

Validation, conceptualization, case management,: Mina Sadat Mirshoja; Methodology, editing and finalization: Ali Akbar Pahlavanian; Drafting: Reyhaneh Najarian; Research: Sadegh Adabi.

\section{Conflict of interest}

The authors declared no conflict of interest.

Acknowledgment

We would like to thank the Student Research Committee and the Neuromuscular Research Committee of Semnan University of Medical Sciences. 
This Page Intentionally Left Blank 
مقاله مرورى:

\title{
شيوع رخداد افتادن و عوامل مرتبط با آن در سالمندان ايرانى: مرورى نظاممند بر مطالعات كَذشته
}

\author{
على اكبر يهلوانيان' هـ ريحانه نجاريان"، صادق ادبى" "مينا سادات ميرشجاع' هـ \\ ا. مركز تحقيقات توانبخشى عصبى عضلانى، دانشكده توانبخشى، دانشكاه علوميزشكى سمنان، سمنان، ايران.

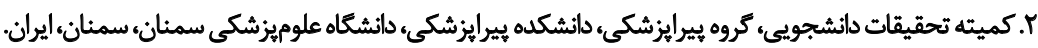

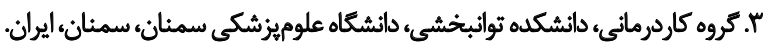

\begin{abstract}
حكיد

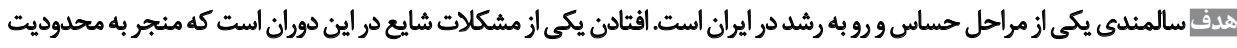

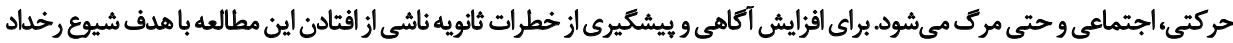

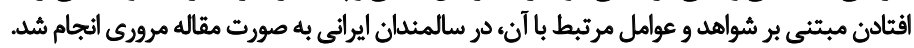

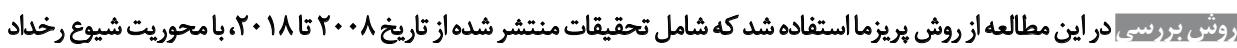

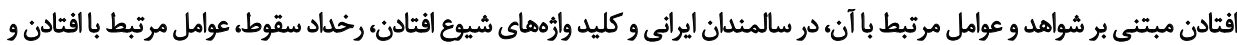

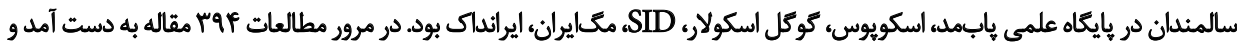

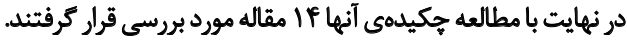

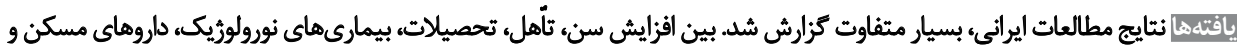
ايمنى مئزل ارتباط معنادار ديده شيد.

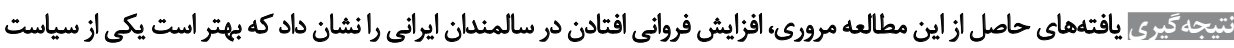

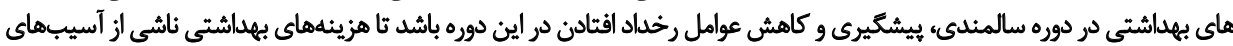

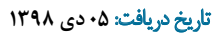

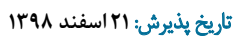

تاريخ انتشار: •1 مهر

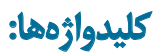

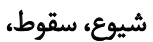
سالمنداني ايران سوط،

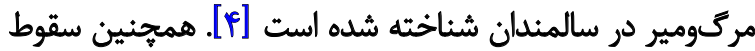

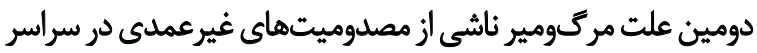

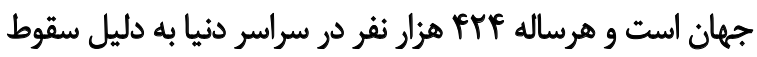

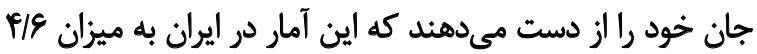

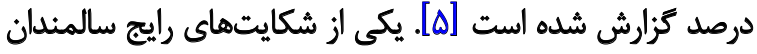

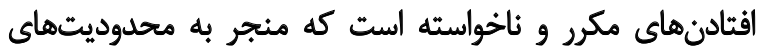

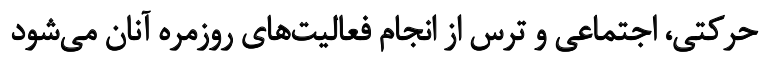

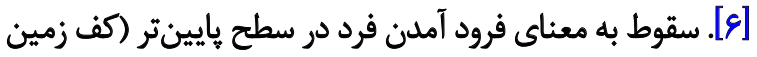

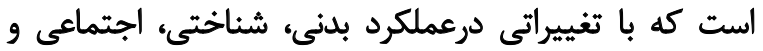

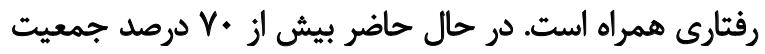

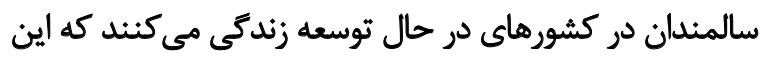

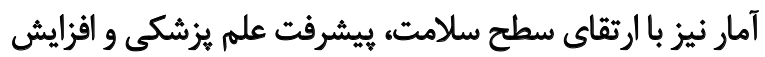

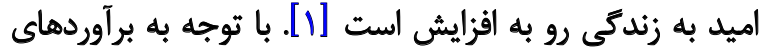

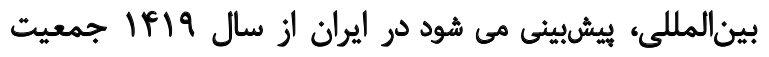

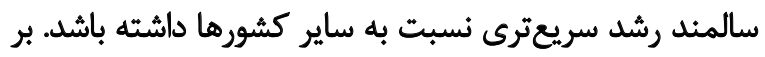

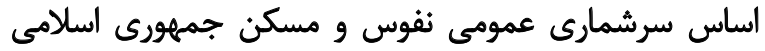

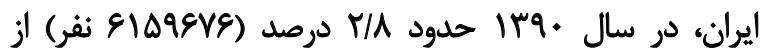
يا روى يك شىء) همراه با آسيب جسمانى و روانشناختى فردئ استر

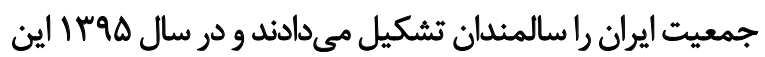




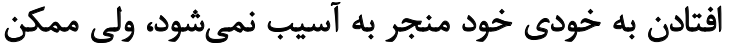

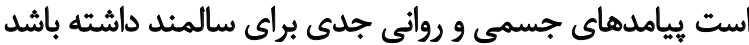

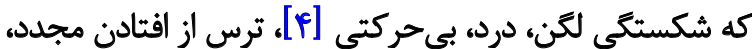

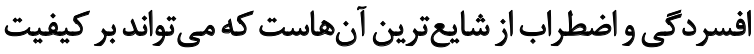

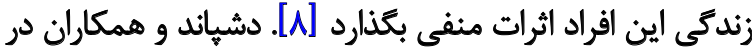

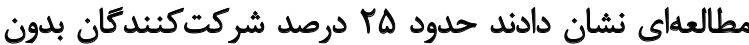

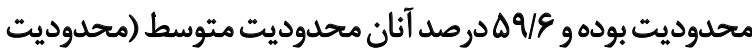

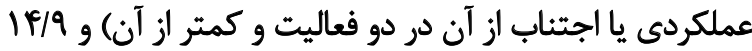

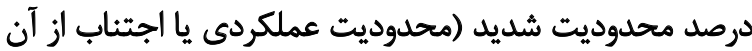

در سه فعاليت يا بيشتر از آن) دارند [بحداد].

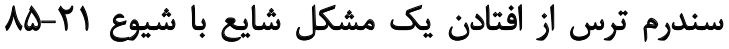

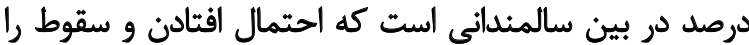

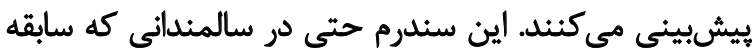

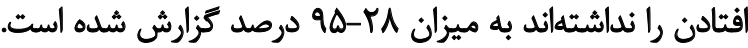

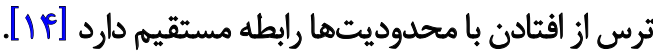
سازمان بهداشت جهانى بيان كرده است كه دادههاى مقطعى

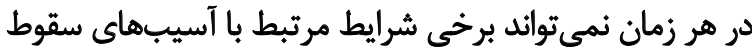

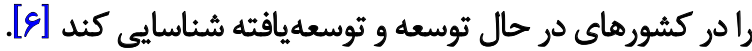

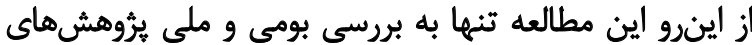

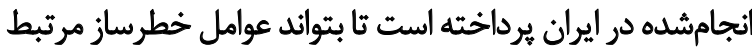

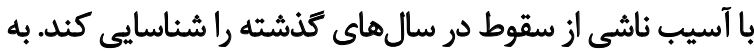

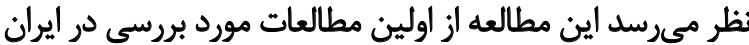

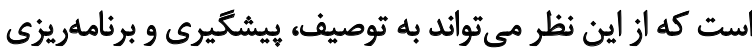

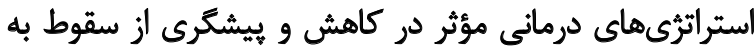

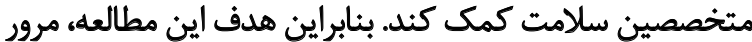

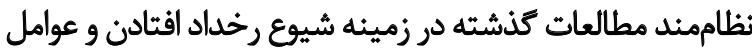
مرتبط با آن در سالمندان ايرانى بود.

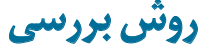

در اين مطالعه از روش يريزما كه جكونكي كزارش استاندارد

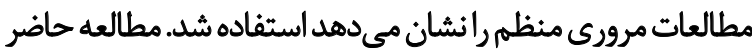

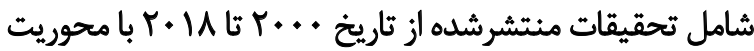

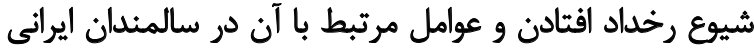
بود كه با استفاده از كليدوائههاي شيوع (Prevalence) و نهانه

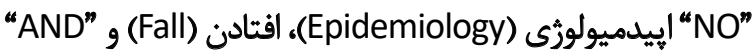

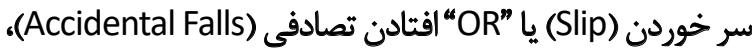

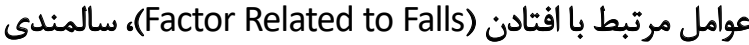

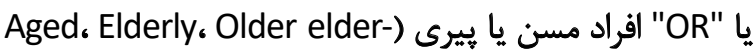

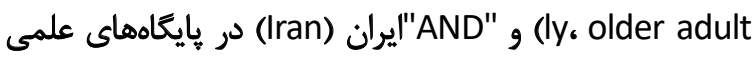
يابمد، اسكويوس، كوكل اسكولار، مركز اطلاعات علمى جهاد

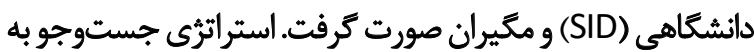

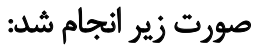

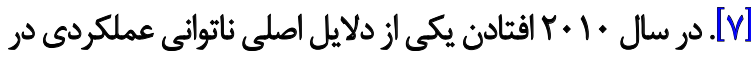

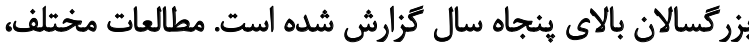

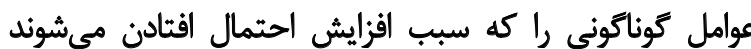

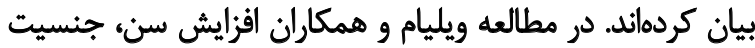

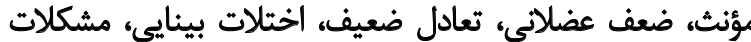

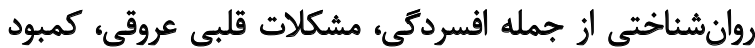

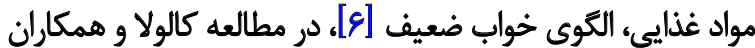

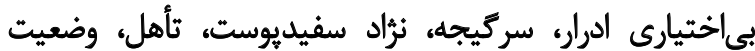

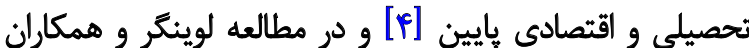

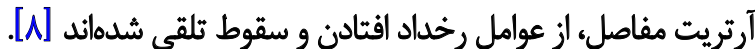

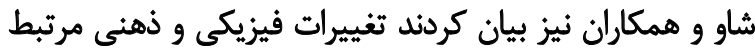

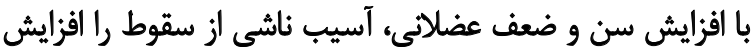

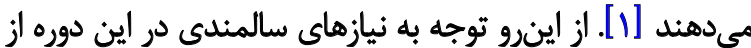

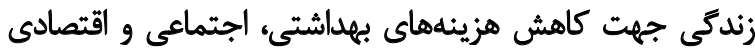

$$
\text { امرى ضرورى به نظر مىرسد. }
$$

مطالعات نشان دادهاند يكي از آسيبهاي اجتماعى و اقتصادى

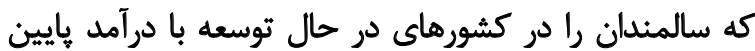

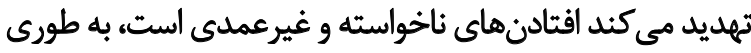

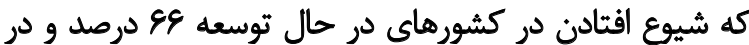

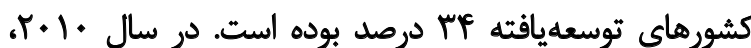
نفر

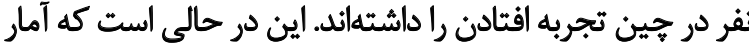

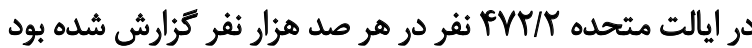

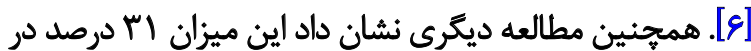

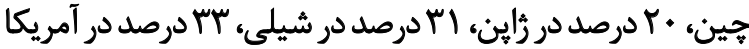

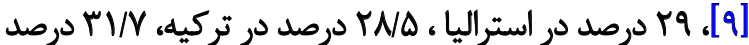

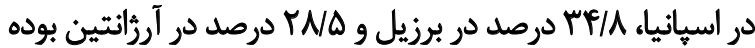

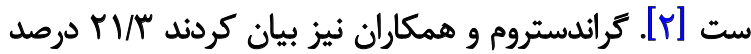

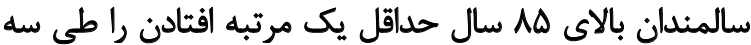

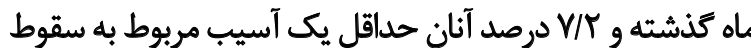

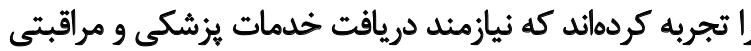

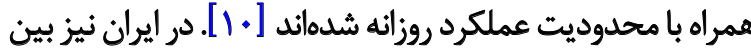

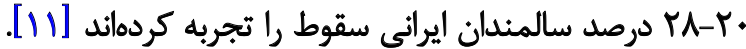

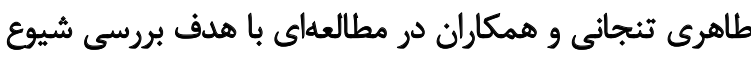

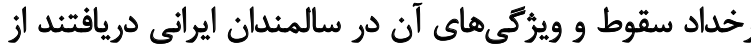

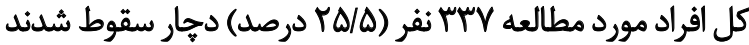

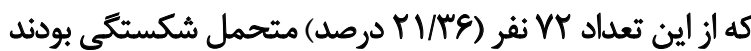

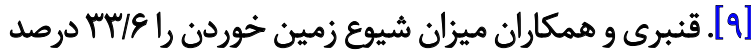

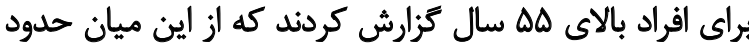

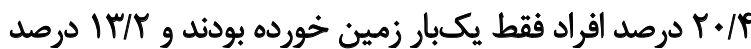

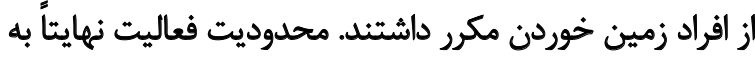

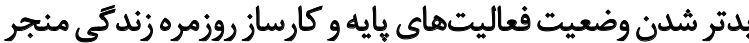

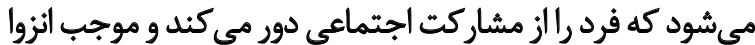

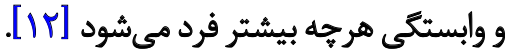




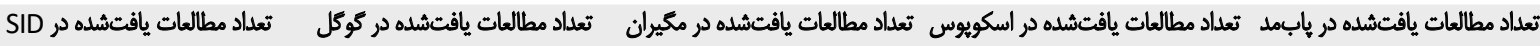
$(n=1)$ (n=1rq) سكولان $(n=r)$ $(n=r \Delta)$

$\downarrow$ تعداد كل مطالعات (n=IVT)

$\downarrow$

(n=10) تعلاد هطالعات خاتجشده به علت در دسترس نيودن متن كامل تعلداد مطالعات خارجشه به علت تكرارى بودن (n=Yه)

$\downarrow$ (n=ITV) تعلاد مطالعات باقيماندانه

$\downarrow$

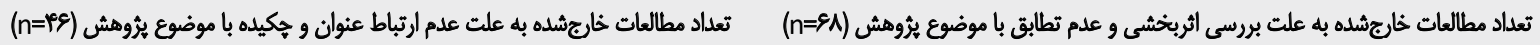
$\downarrow$

تعداد مطالعات بررسىشده (n= (n)

تصوير ا. مطالعات جستوجوشده در مراحل غربالكرى، احراز ملاكهاى ورود به مطالعه شيوع رخداد افتادن و عوامل مرتبط با آن در سالمندان ايراني توانبخننى ترسه

كزارش كردند [9]. نعمانى و همكاران فراوانى تعداد دفعات زمين

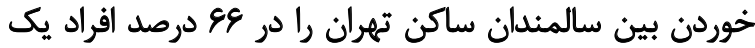

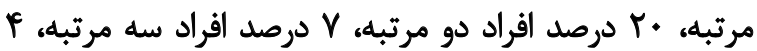

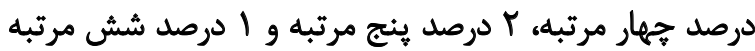

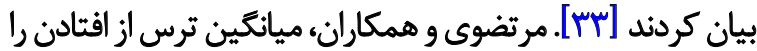

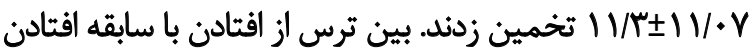

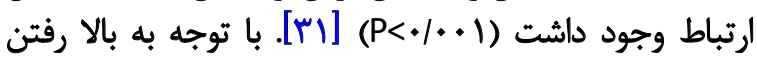

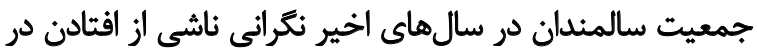

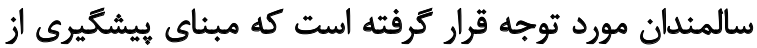

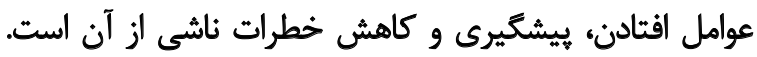

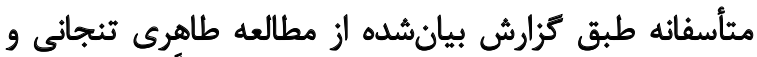

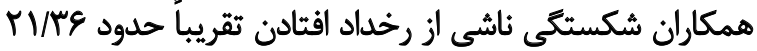

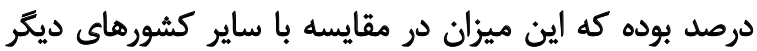

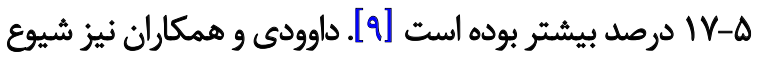

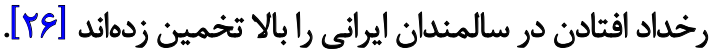

مرعشى و همكاران نشان دادند ميزان سقوط در كروه دروه

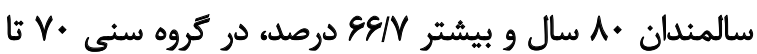

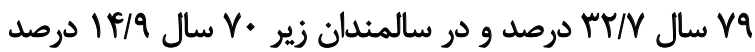

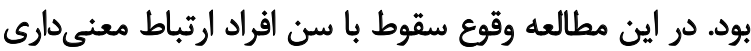

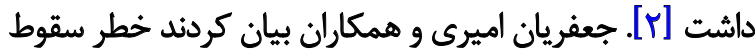

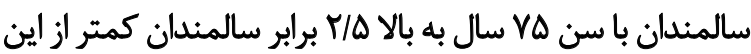

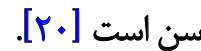

قنبرى و همكاران در برؤهش خود كزارش كردند كه سقوط

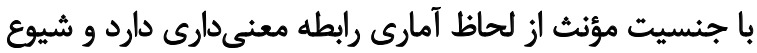

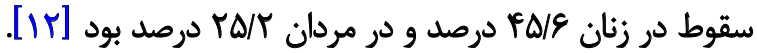

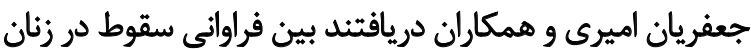

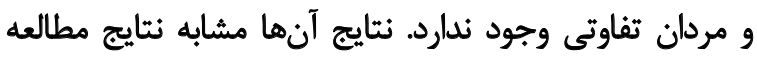

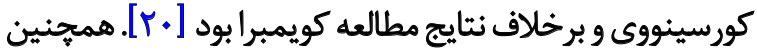

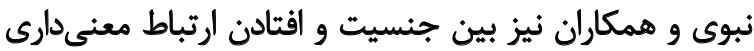

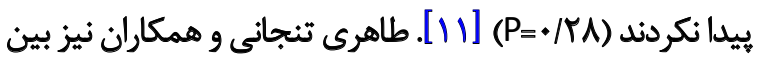

("Prevalence") NO ("Epidemiology") AND ("Fall" AND "Slip" OR "Accidental Falls" AND "Factor Related to Falls") AND ("Older Adult" OR "Older" OR "Older elderly" OR "Elderly" OR Aged") AND "Iran" ملاك هاى ورود به مطالعه براى تحقيقات فارسىزبان و لاتين

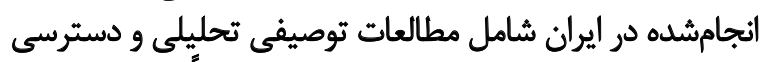

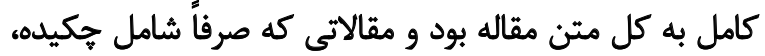

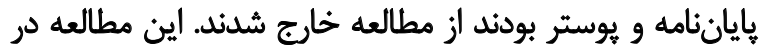

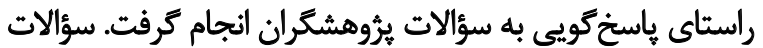

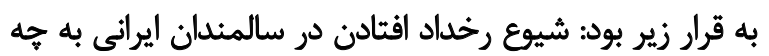

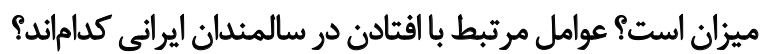

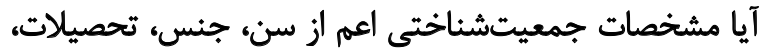

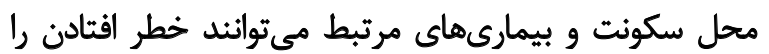

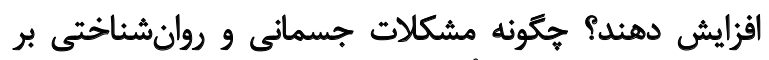

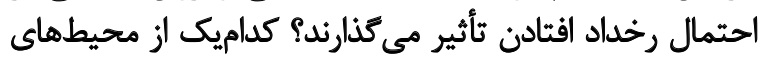
خانه احتمال افتادن را افزايش مي دهندي؟

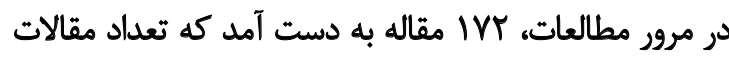

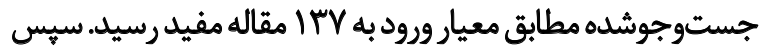

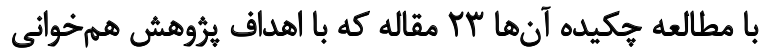

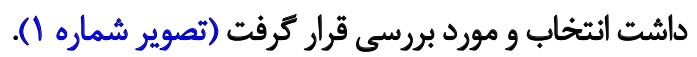

ياقتهنها

در اين مطالعه هريك از تحقيقات مرورشده به اختصاردر جدول

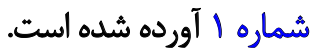

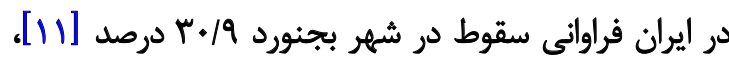

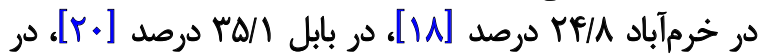

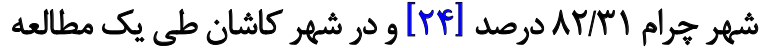
كذشتهنكر جهارساله

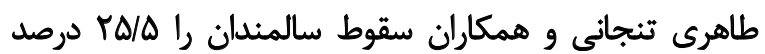


جدول ا. نتايج بررسى متون كذشته در بررسى شيوع رخداد افتادن و عوامل مرتبط با آن در سالمندان ايرانيى

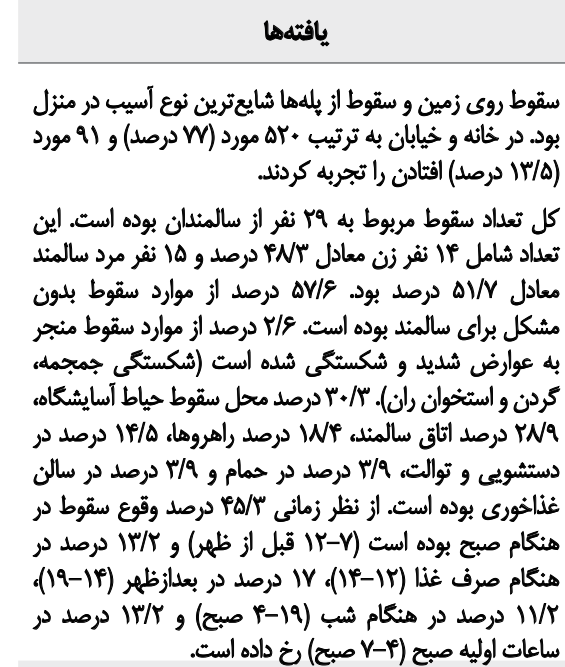

اين تحقيق ششان داد بيمارى هاى مزمن، خطر زمين افتادن رادر سالمنلان به طور جشمكيرى افزايش مى دهنئ.

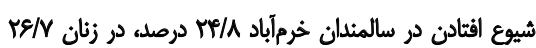

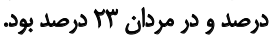

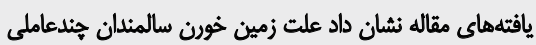

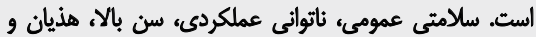

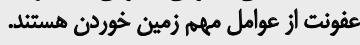

اقلامات بيشكيرى الز سقوط در و و دروصد در حل خوب و در

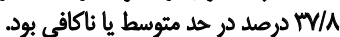

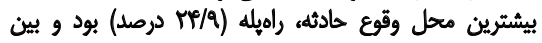

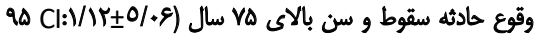

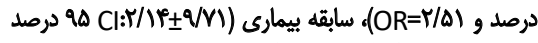

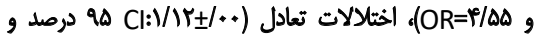

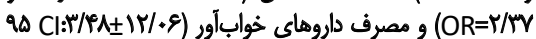

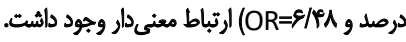

يش از •ب درصد از افراد بالاي هو سال حلاقل يكبار در سال

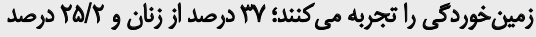

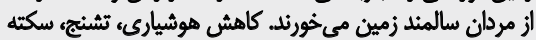

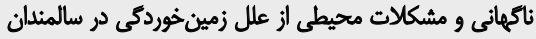

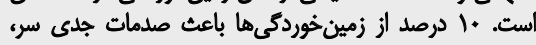

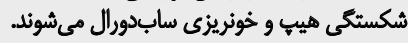

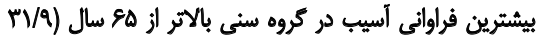

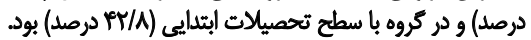

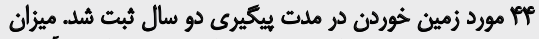
زمين خوردن

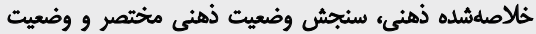

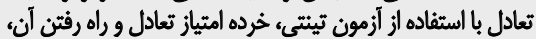
وضعييت أفسردكى سالمندان

\begin{tabular}{|c|c|c|c|}
\hline تيستهاي ارزيابي & تعداد نمونه و روش اجرا & عنوان & نام نويسئده \\
\hline $\begin{array}{l}\text { مقياس مصدوميت كوتال } \\
\text { (AIS-90) }\end{array}$ & مطالعه مقطعى روى PVD سالمند & جراحات ناشى از باييز در & 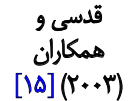 \\
\hline
\end{tabular}

تحقيق از بيمار،

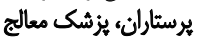
و يروندهاى بيماران يزان مطالعه مقطعى روى Vع مورد

ويرّكى هاى زمين منوردن

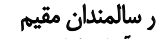
آسايشكاه

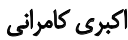
it [iv] (r..V)

\begin{tabular}{|c|c|c|c|}
\hline 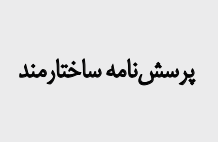 & 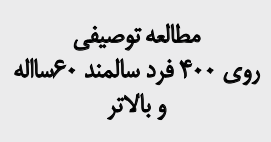 & 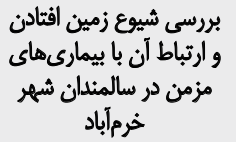 & 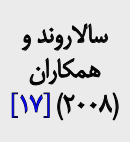 \\
\hline 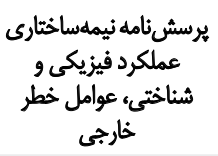 & يك مطالعه توصيفى مقطعى مالمند & زمين افتادي دور سالمندان بان & 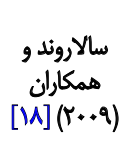 \\
\hline سلامتى عمومى، ناتواني & 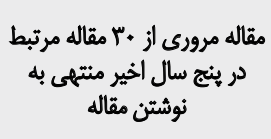 & 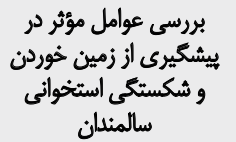 & 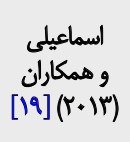 \\
\hline
\end{tabular}

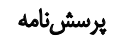

يثروهشكرساخيّه جمعيتششناختى مشخصات مربوط بهاته وقوع حادثه و اققدامات

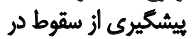
مثنل (در هد خدوبي متوسط و ضعيف) شامل اطلاغات

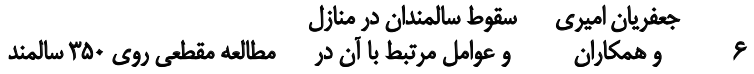

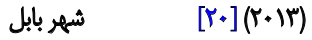




\section{بافتهن}

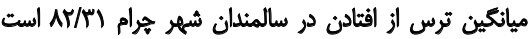

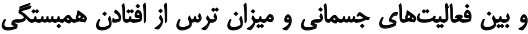

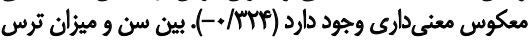

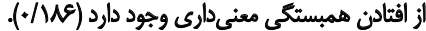

ميانكين سابقه سقوط حداقل يك مرتبه در سالمندان س/ TV درصد

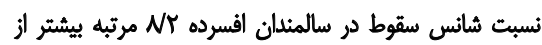

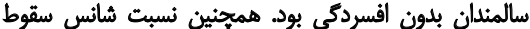

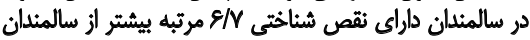

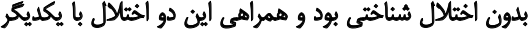

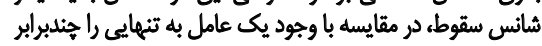
مي كرد.

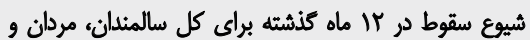

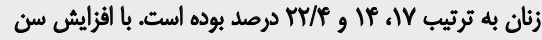

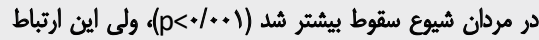

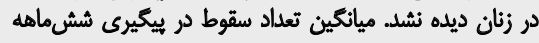

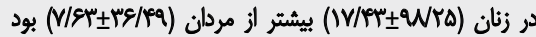

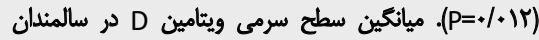

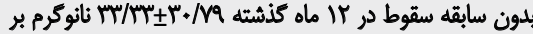

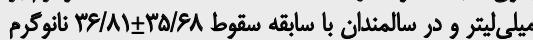
بر ميلى ليتر بوده است.

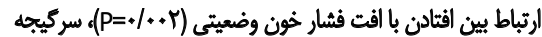

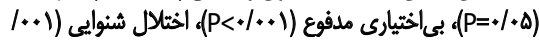

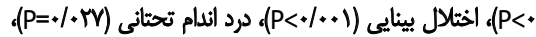

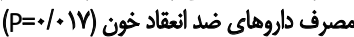

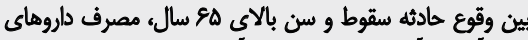

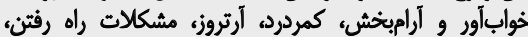

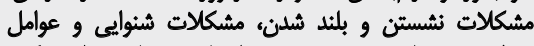

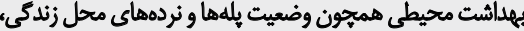

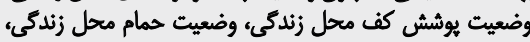

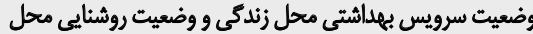

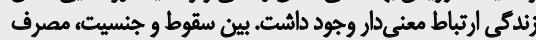

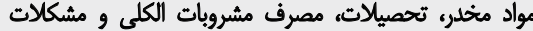

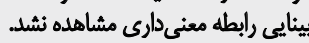

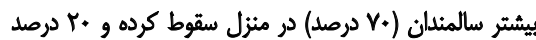

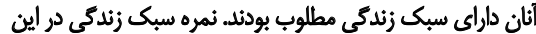

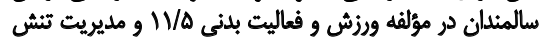

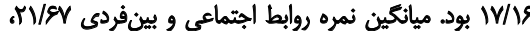

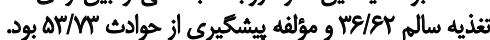

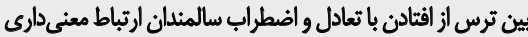

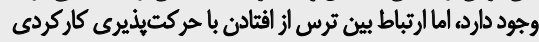
هعنى دار نيست.

تعداد نمونه و روش اجرا تستهاى ارزيابي

كايرسش نامه كارآهدى

افقتادن فرم بين الملماري

(FES-I)

ابزئرهاي اسثائدارد

Easy-Care

تحرى مبتني بر عملكرد (POMA)

وضعيت شناختى از

ي برسشنامه استاندارد

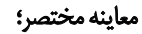

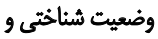

سنجش افسردمى ازي

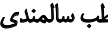

سنجش ويثامين D از

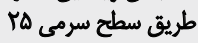

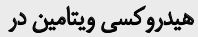

نمونه خون صبحكاهي مينى سالمندلن

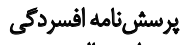

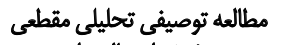

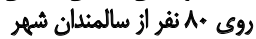
جهرام
برانى ميزان ترس الز

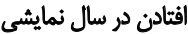
شهير ثرام نامي

1

$[\pi /(T+18)$
ييشيبنى عوامل خطرساز توصيفى تحليلى به صورت

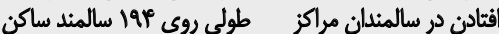
كهريزك تهران نكهبارى

شريفى و مارن | $[T \Delta](T+10)$

يك مطالعه مورد شاهد (  (ij,

إراسى ارتباط الفسردمى و

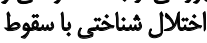
منجر به شكسئكى در سالمثلان

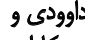

ir

$[r \varepsilon](T \cdot 18)$

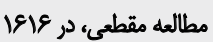

فالمند شهر اميركانلا

فراوانى سقوط طيى دو مرحله بررسى شلد. در مرحله اول در مر درو

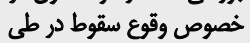

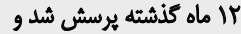

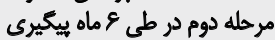

شيوع سقوط و همراهيى آن با سطح سرمى مرامي ويثيامين Dر مان سالمندان شهر اميركلا

همينى و II همكاران سالمثلنان أنجام كرفت.

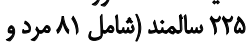

إبرات ناميه شامل

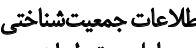
و عواهل مرتبط بازمين خوردن
18. مطالعه مورد شاهدى روى سالمند سالماهن

4

عوامل مرتبط بازمين خوردن در سالمثلدان مبتالا

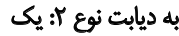
هطالعه هورد شاهدي دري سالمثدان مراجعه كنثله به درد بيمارستان

\section{هم: If [rA] $(T+19)$}

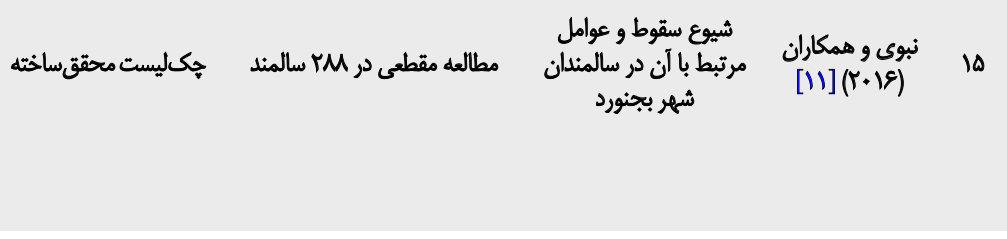

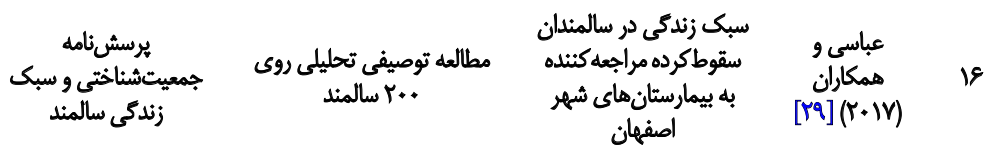

يربشئنامه اطلاعات كرادي، بردسش فامه كار أمدى افتادن فرم بين المللى، مقياس فراس درجهبندي اضطراب هميلتون، أزمون تعادل

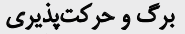
كاركردى توسط آزمون

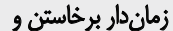
رفتن، +" ثاثيه برخاستن و ونشستن برن 


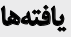

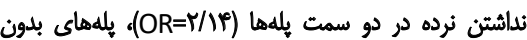

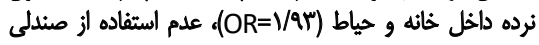

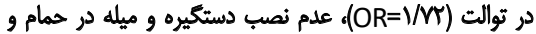

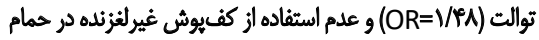
و توالت (OR=//TI) به عنوان موامل خطر سقوط سالمندان شنايسايى شدند.

ارتباط معنى دار بين ايمنى مثنل و ترس از اقتادن در سالمثدان $(\mathrm{P}</ 1 \cdot \cdot 1)$

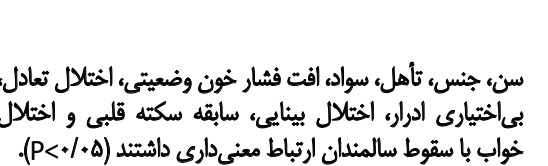

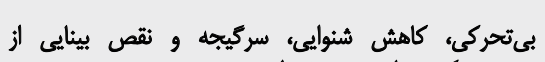

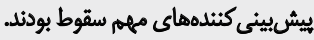

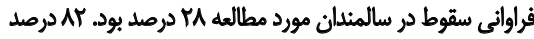

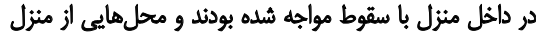

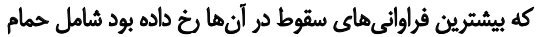
W) (IV) درصد) و يذيرايع (9 ا درصد) بود.

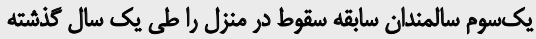

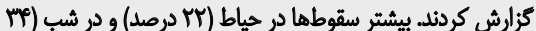

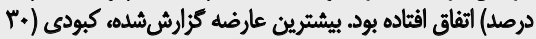

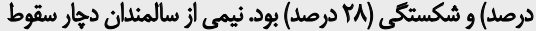

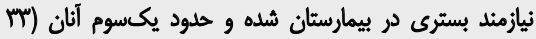

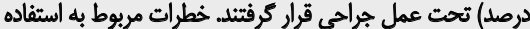

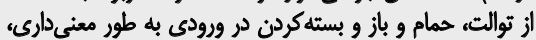

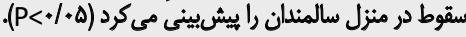

تعداد نموثه و روش اجرا تستهاى ارزيابى

عنوان

رديف نام نويسنده

خاثكاليست فضايى خائه محل زندكى سالملي وحياط يربش ثامه كارأمدى إقتادن فرم بين المللى (FES-I)

\section{هيرسشنامه \\ شامل اطلاعات}

جمعيتش شئاختيى، سؤالات مريوط به مشخصات وقوع سقوط در يك سال كنتشئه و برخى عوامل فردى هرتبط با سقوط ئماط

\section{برسشئامه}

جمعيتشناختى، رفتارى، جسمائى

يرسش ئامه محقق ساخته

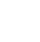
محيطى و بيمارى هناي

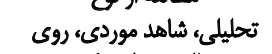

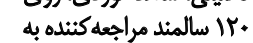
بخش هاي اورئانس مراكز أموزشى درمائى با علث سقوط التر

خطرات فضاى فيزيكي

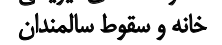
ايرانفر و همكاران $[\Delta](r+1)$

يكى مطالعه مقطعى روى +Pa سالمثلد

Thent

مطالله مقطعى )وى A ع صاله

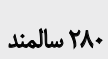

(1)
مطالعه توصيفى تحليلي به به موري

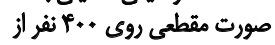 سالمندان شيهر تئران

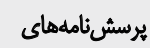
جمعيتشناختى و غربالكرى خودئزارشى سقوط وحوادث در مثزل Home Fast-SR
مطالعه توصيفى مقطعى روى ساكن شهر قزوين

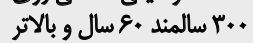

ارتباط بين امنيت خانه و شييوع ترس از افتادن در ميان سالمنلان: يكى هطالعه مقطعى

برزسى رخدلاد سقوط

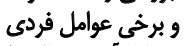
مرتبط با آن در سالمثدان مراجعه كنثلده به بائيكاهماي سبلامت تحت يونش برت شبكه بهيدانست و ودرمان شهرستان رى در سالل رستان

عوامل خطر سقوط در بزركسيالان سالمند أيرائى:

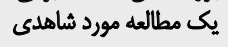

بررسي فراوائى عوامل

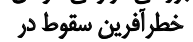
سالمندان كيرنده خخدمات درمانى در مئزل در سال Irge

هرتضوى 29 [ri ] $(r+\mid k)$

مرعشى و r.

[r] $(T+1)$

طاهرى و M [Tr] (r.19)

نعمانى همكاران [rit] $(r \cdot 19)$

شيوع سقوط در منزل و و

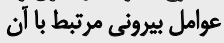
در سالمثدان شهر قزئن مرين

باقرى روجى Th $[r+r \cdot r \cdot)$

توانبخننى

عباسى و همكاران [1 [ ] نشان دادند سقوط در سالمندان كمبسواد

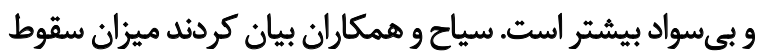

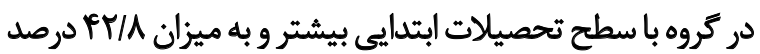

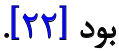

در مطالعه مرعشى و همكاران، رابطهاي بين تأهل و سقوط

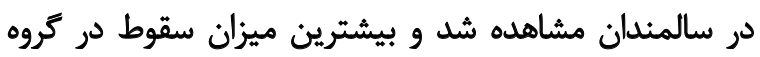

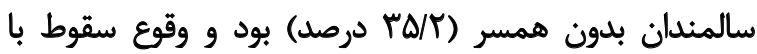

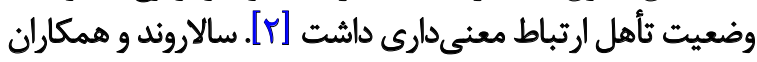

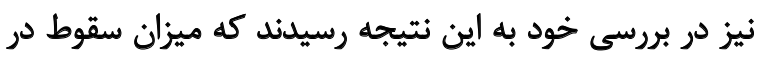

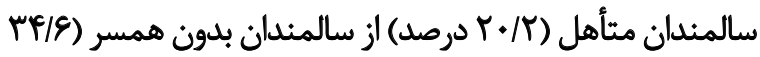
درصد) كمتر است و سقوط با تأهل ارتباط معنى دارى دارد [ [IA]

الواوودى و همكاران نشان دادند شانس سقوط در سالمندان

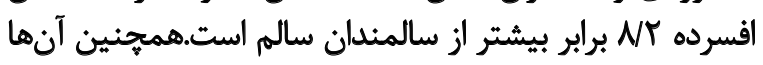

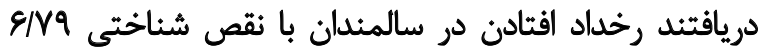

و افتادن سن رابطه معنى دارى نيافتند، اما كزارش كردند مئد ميزان

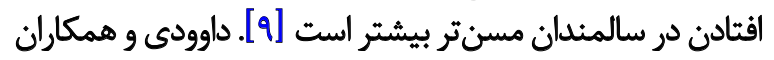

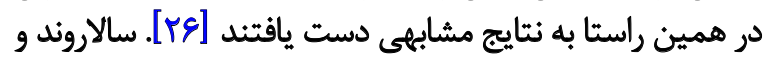

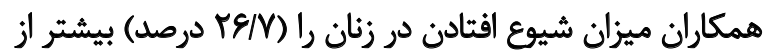

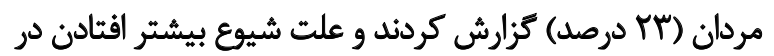

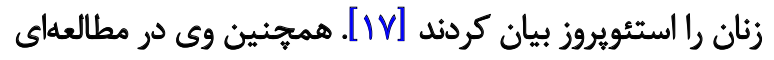

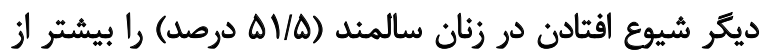

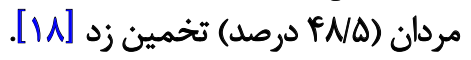

صلواتى و همكاران ارتباط معنى دارى بين سطح تحصيلات

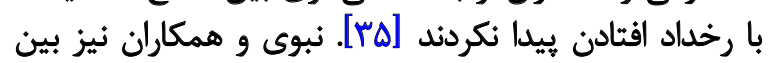

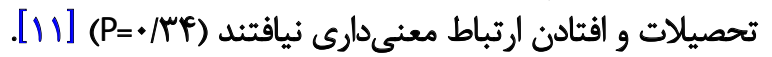

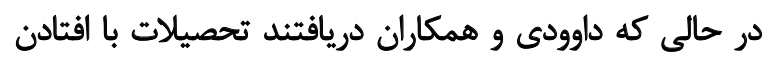

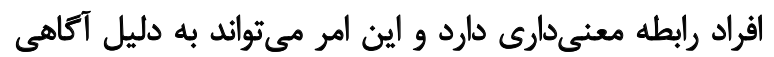

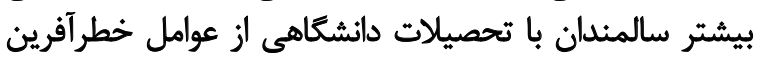

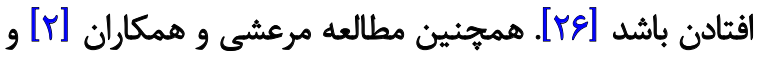




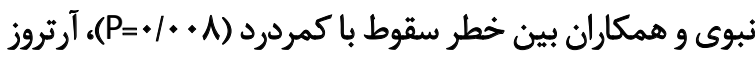

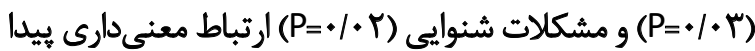

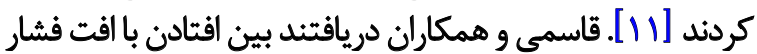

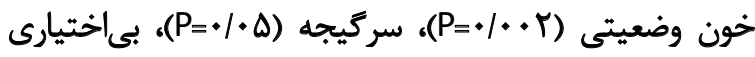

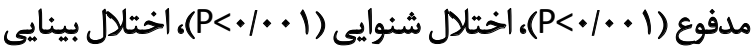

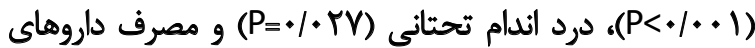

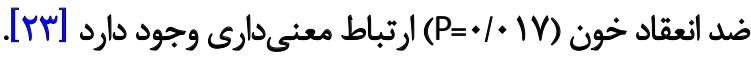

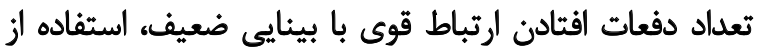

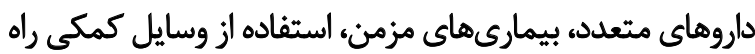

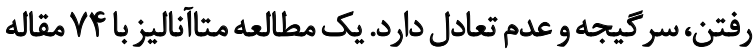

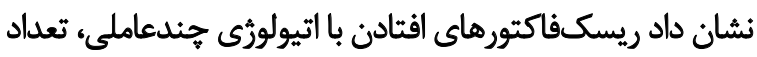

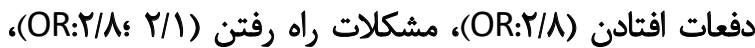

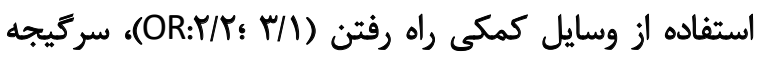

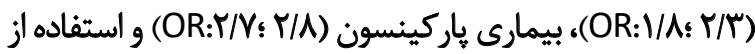

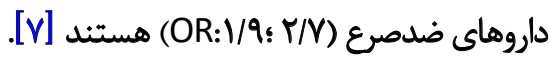

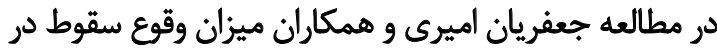

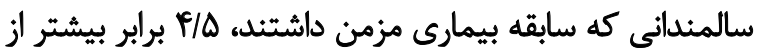

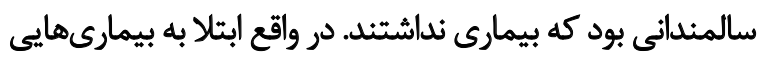

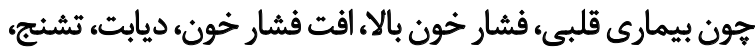
سردرد و سركيجه، بيمارىهاى استخوانى و مفصلي، اختلال تعادل و راه رفتن و مشكلات بيناييى در سالمندان مى تواند منديج

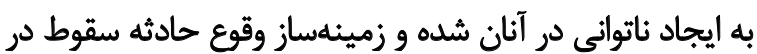

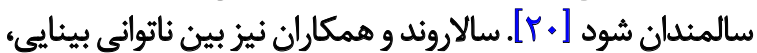

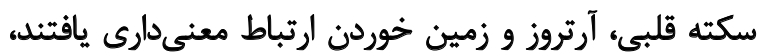

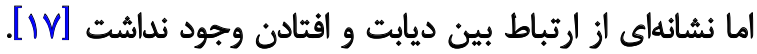

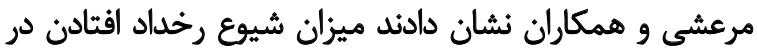

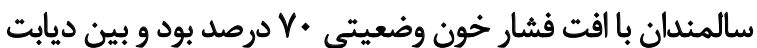

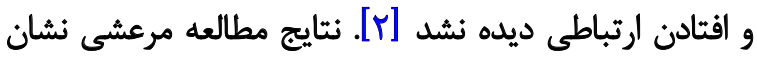

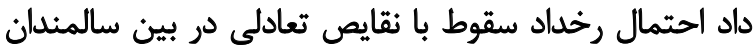

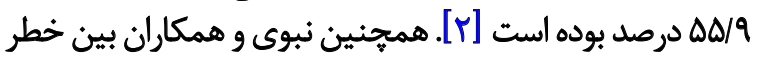

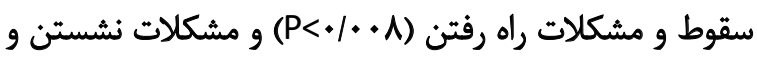

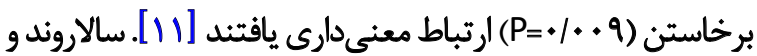

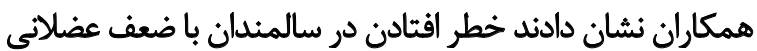

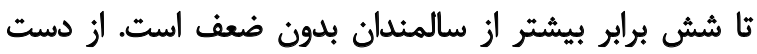

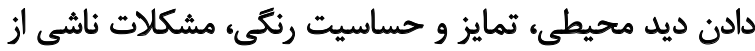

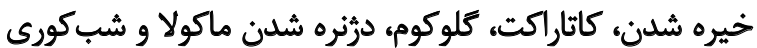

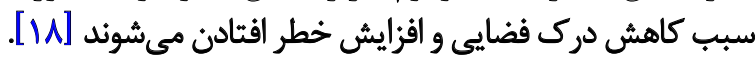

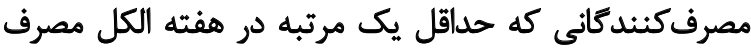

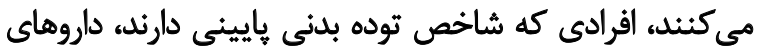

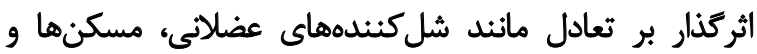

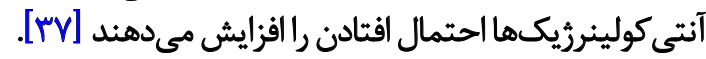

جعفريان اميرى و همكاران دريافتند ارتباط معنىارى بين
برابر بيشتر از سالمندان بدون نقص شناختى بود كه علت آن

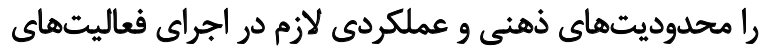

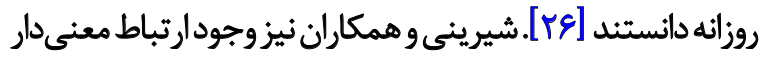

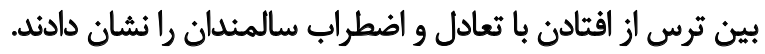

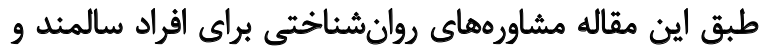

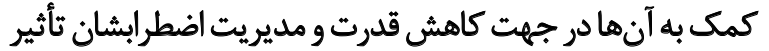
بسزايى در افزايش تعادل آنها دارد [ـــــ

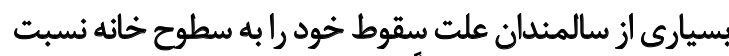

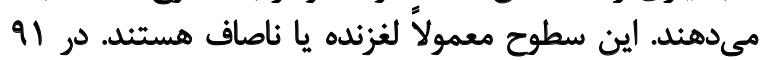

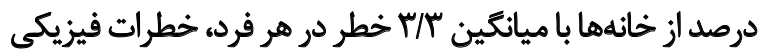

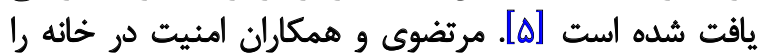

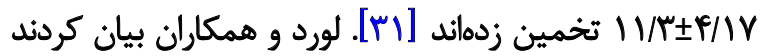

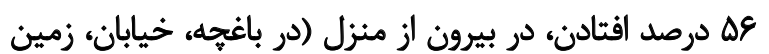

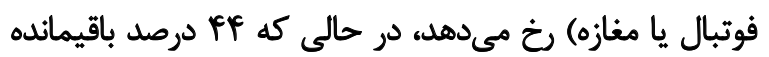

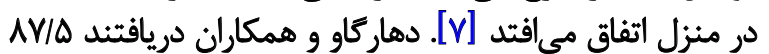

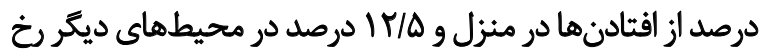

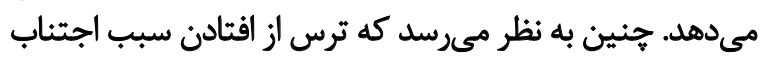

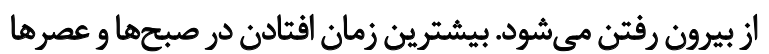

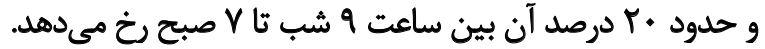

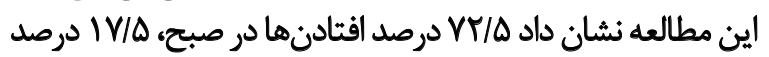

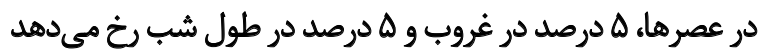

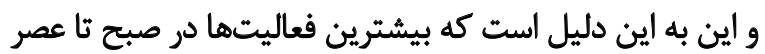

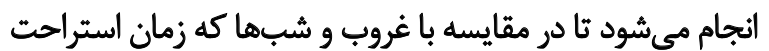

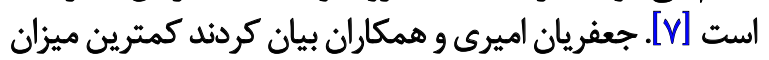

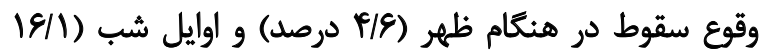

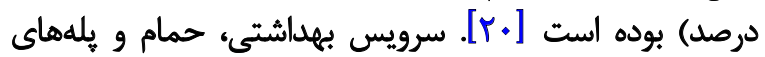

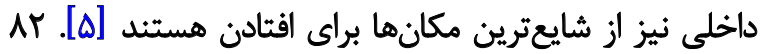

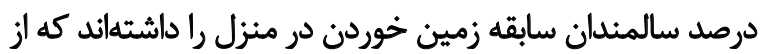

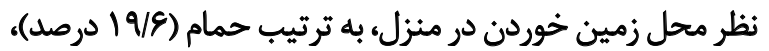

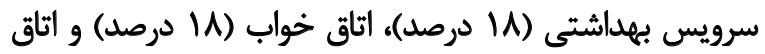

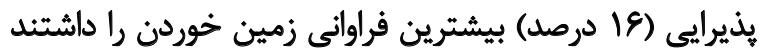

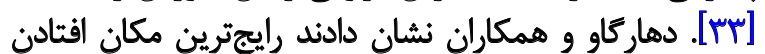

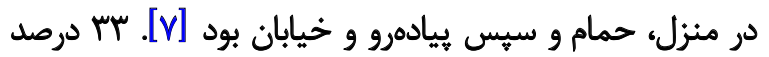

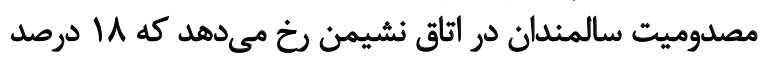

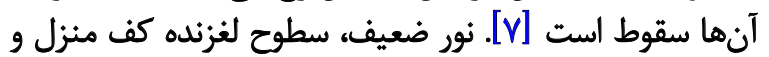

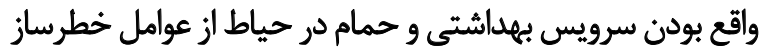

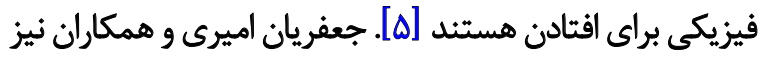

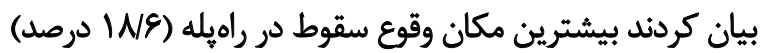

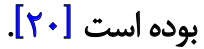

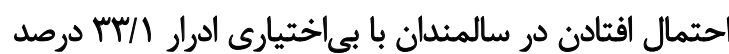

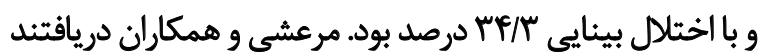

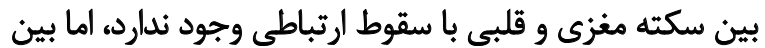

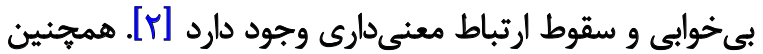


جدول r. عوامل مرتبط با افتادن در سالمثدان ايرانى

\begin{tabular}{|c|c|c|}
\hline نامطلوب & مطلوب & عوامل مرتبط با اقتادن \\
\hline is & هرد & جنسيت \\
\hline مسنتر & 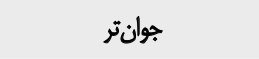 & سن \\
\hline مجرد، بيوه، مطلقه & متأهل & ت ت ت أهل \\
\hline درآمد بايين & درأمد متوسط و رو به بالا & الشتغال و سطح اقتصادى \\
\hline سطح تحصيلات بايينتر & سطح تحصيلات بالاتر & تحصيلات \\
\hline مقيم در مراكز نكهدارى & غيرمقيم در مراكز نكههارى & محل سكونت \\
\hline ثاسالم و دركير در بيمارى & 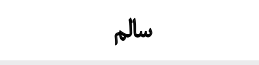 & بيمارى هاى روانششاختى \\
\hline ثاسالم و دركير در بيمارى & سالم & بيمارى هاى جسمانى \\
\hline
\end{tabular}

جدول ب. دستلبندى عوامل مرتبط با زمين خوردن در سالمندان ايرانى

\section{Laia}

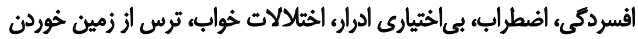

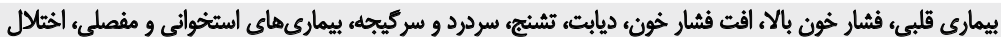

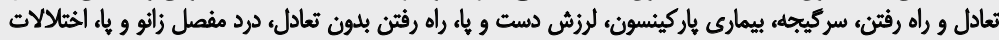

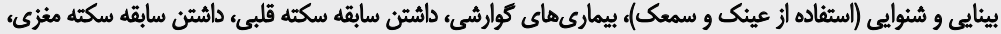

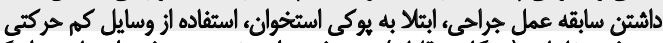

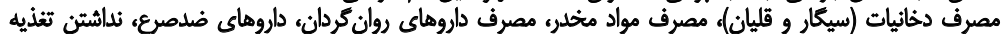

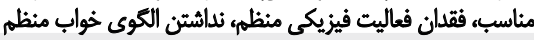

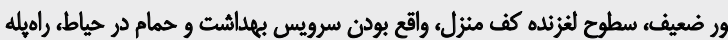

عوامل مرتبط با اقتتادن

عوامل روان نشنائتَى

عوامل بيماري هاي جسماتى

عوامل خطر ساز سبك زندكى عوامل خمطرساز منزل
سازمان بار آسيبهاي غيرعمدى در كشورهاى در حال توسعه

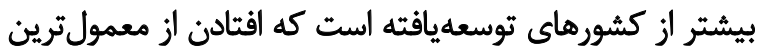

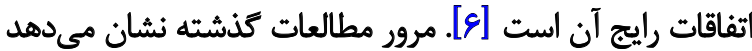

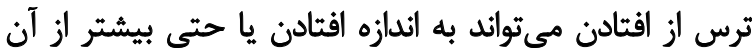

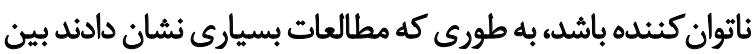

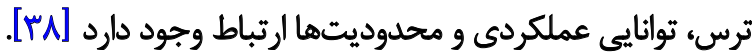

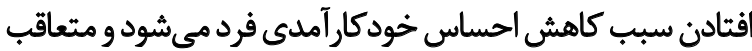

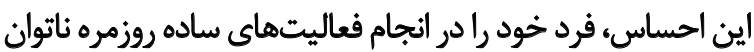

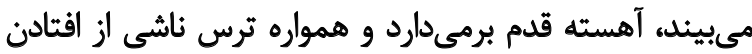

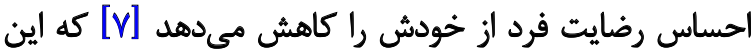
مسئله كيفيت زندكى افراد راتحت تأثير قرار مى دهاند. طبق اين مطالعه مىتوان عوامل مؤثر در زمين خوردن را دو

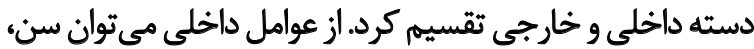

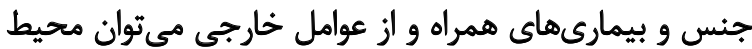
فيزيكى و محل قراركيرى اشياء را نام برد.

شواهد افتادن در بزركسالان مسن بسيار يراكنده و متنوع است.

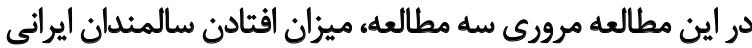

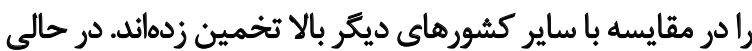
كه نتايج يك مطالعه در ينج شهر ايران نشان دادئ داد فراوانى سقوط

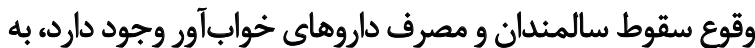

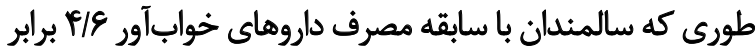

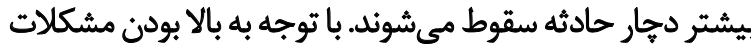

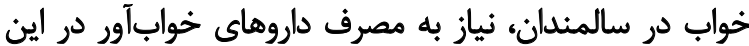

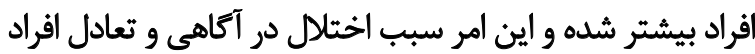

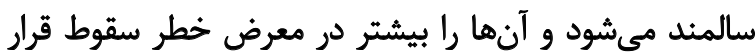

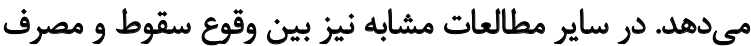

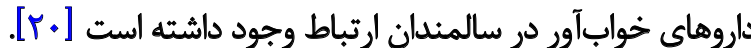
همجنين نبوى و همكاران بين مصرف داروهاى آرامشبخش آردائ

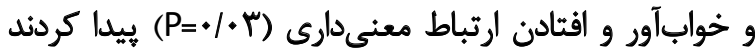

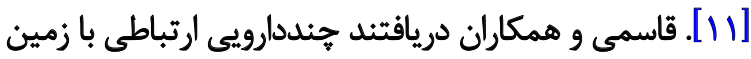

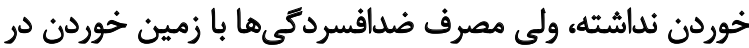

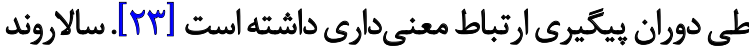

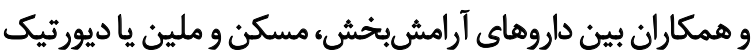
و زمين خوردن ارتباطى وييدا نكردند [11]. بحث

يكى از سياستهاى سازمان بهداشت جهانى بيشكيرى از

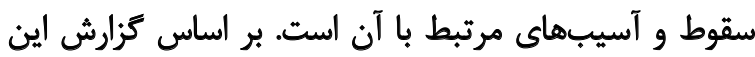


زمين خوردن محسوب شود؛ زيرا به دليل كاهش توان عضلاتى

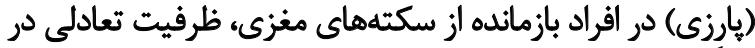

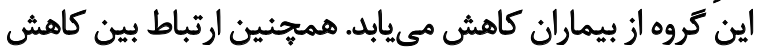

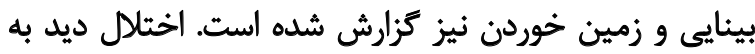

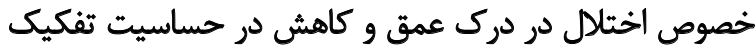

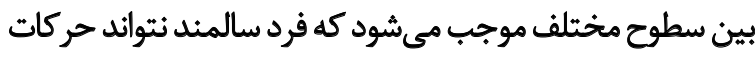

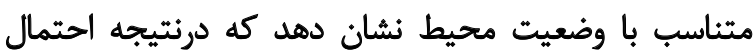

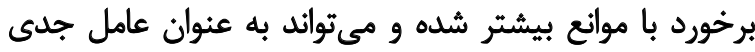

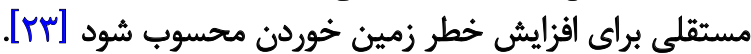

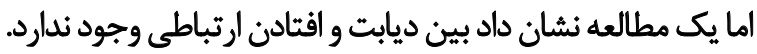

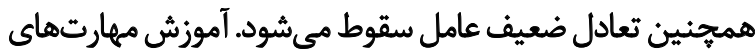

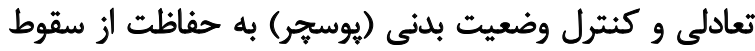

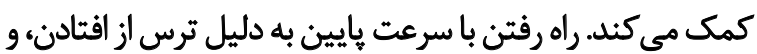

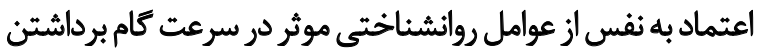

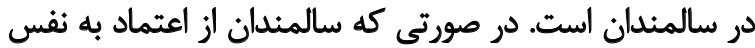

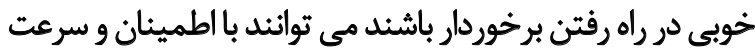

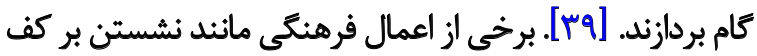

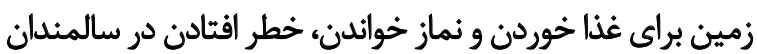

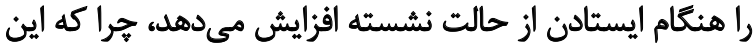

تغيير وضعيت نياز به تعادل و قدرت خوب دارد [لإن]

ينج مطالعه نشان دادند عوامل روانشناختى مانئد افسردكى،

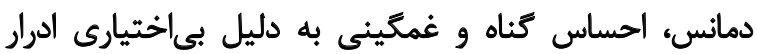

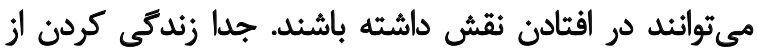

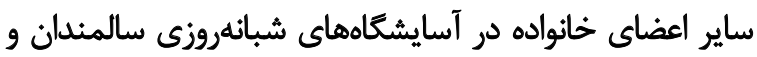

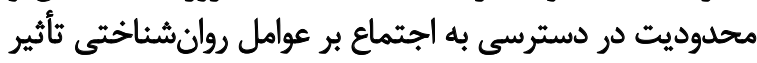

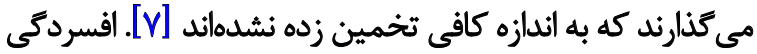

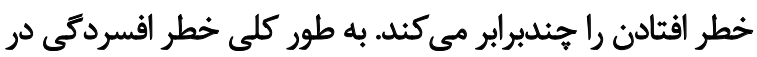

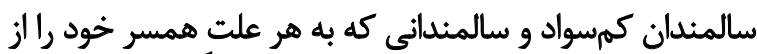

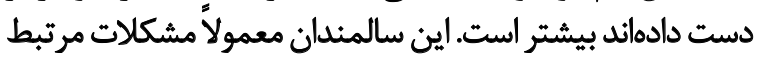

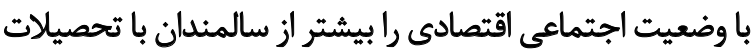

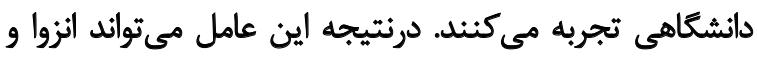

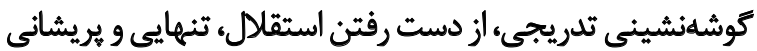

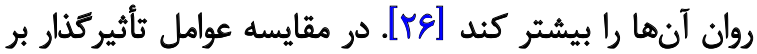

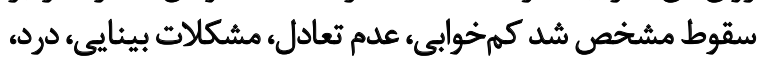

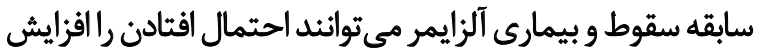

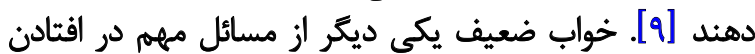

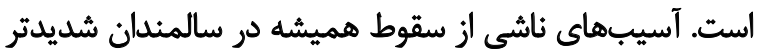

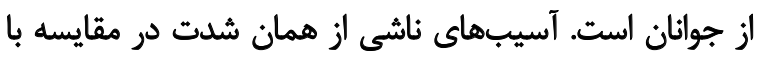

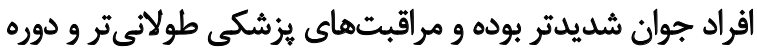

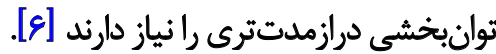

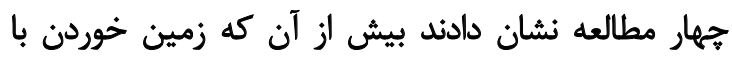

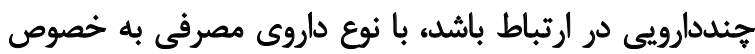

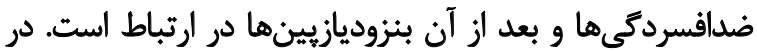

سالمندان ايرائى در دامنه متوسط از آمار كشورهاي ديكّر قرار

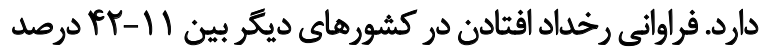

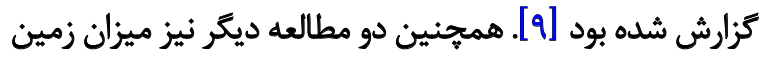

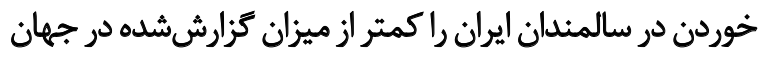

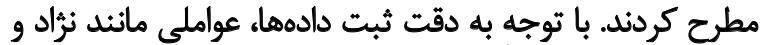

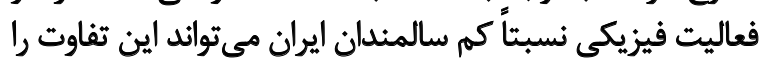

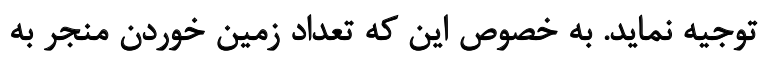

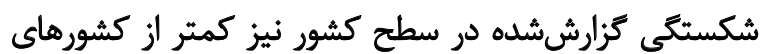

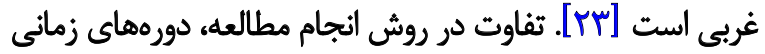

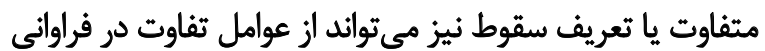

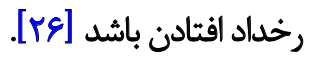

سن يك ريسكفاكتور مشترك بوده است [ع] ـ هشت مطالعه

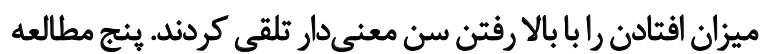

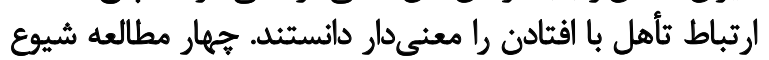

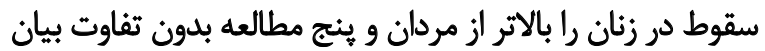

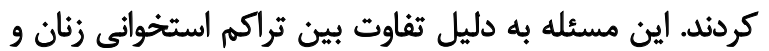

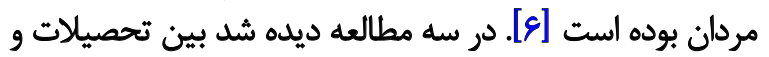

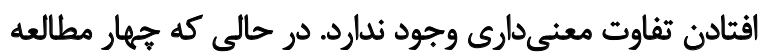

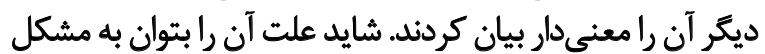

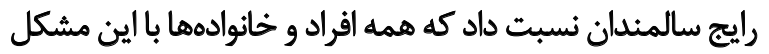

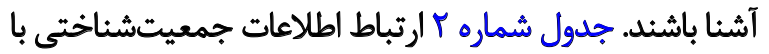
افتادن را به طور خلاصه نشان مى دهد.

آسيبهاى ناشى از سقوط نهتنها بر عملكرد، استقلال و كيفيت

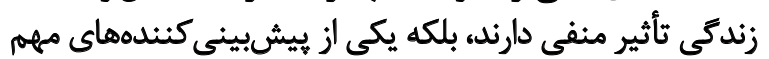

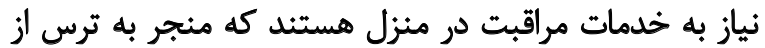

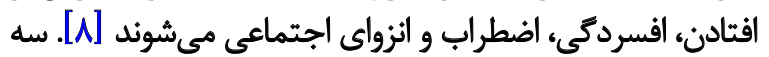

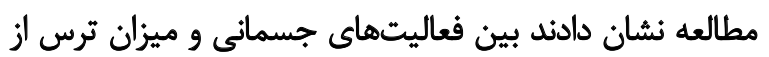

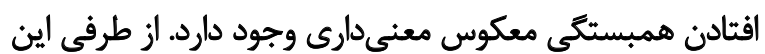

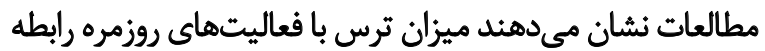

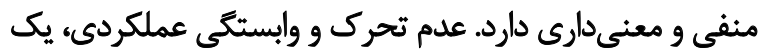

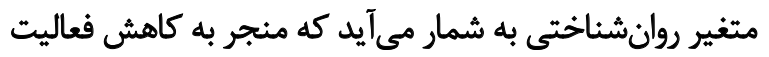

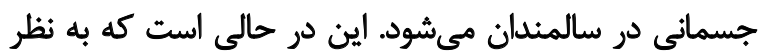

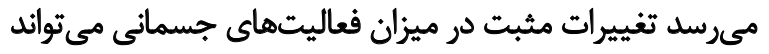

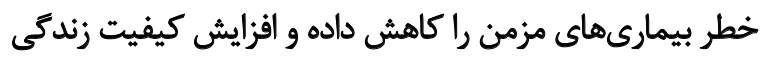

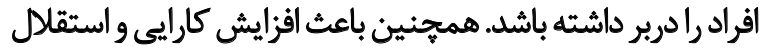

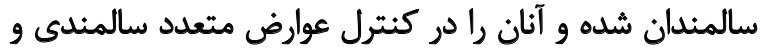

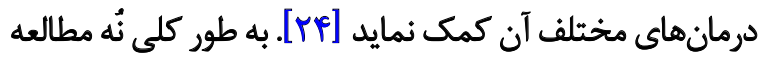

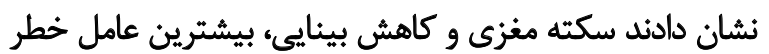

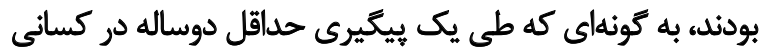

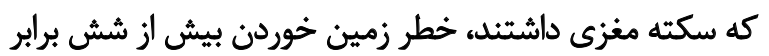

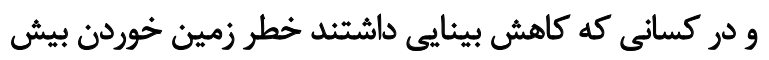

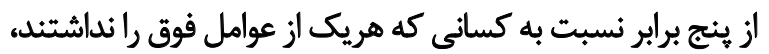
افزايش يافت. سكته مغزى مى تواند به عنوان عامل خيك خطرى برى براي 
ذكر كردهاند [1/]] همجينين از آنجايي كه احتمال افتادن در

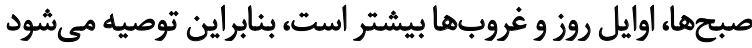

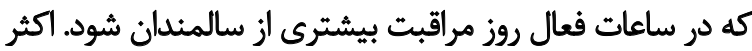

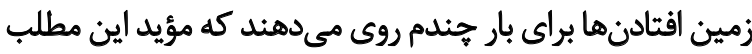

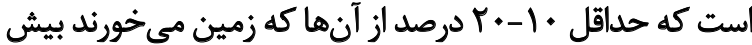

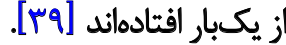

سه مطالعه نشان دادند شكستخى لتن، شكستن دست و ياو

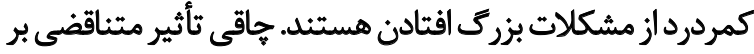

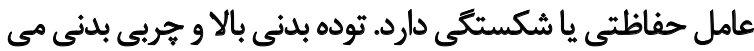

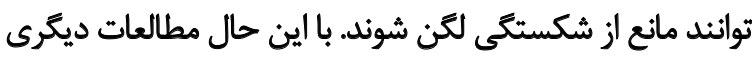

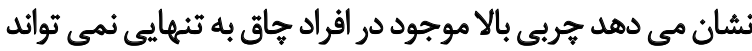

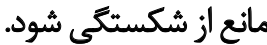

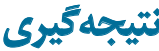

شواهد افتادن در سالمندان بسيار هراكنده و متنوع است.

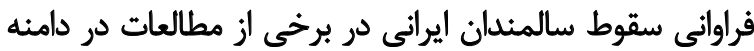

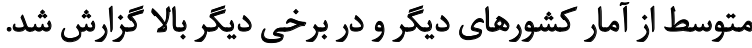

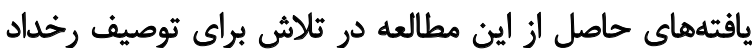

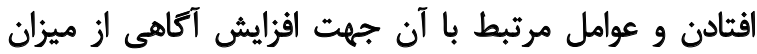

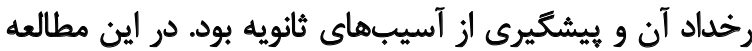

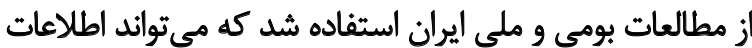

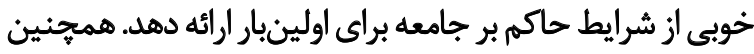

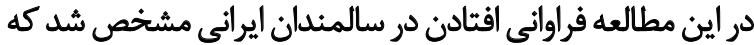

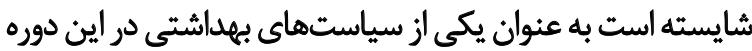

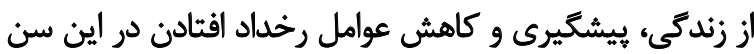

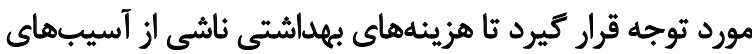

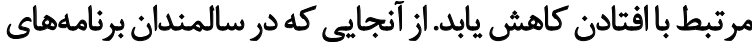

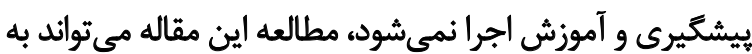

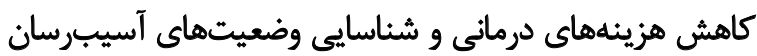

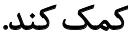

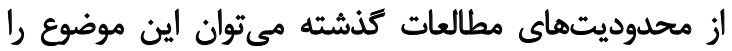

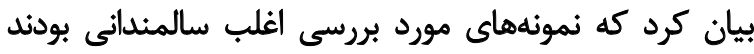

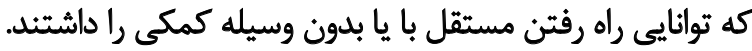

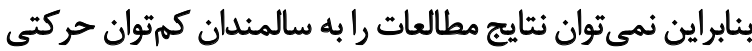

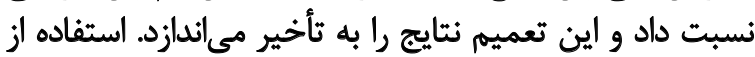

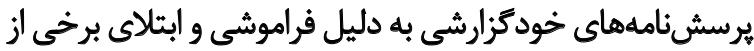

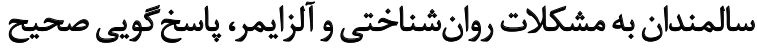

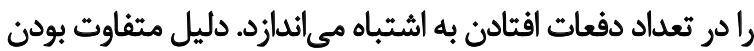

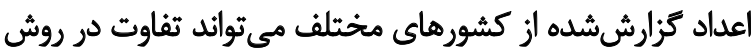

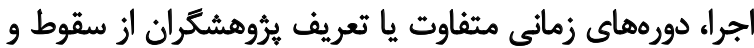

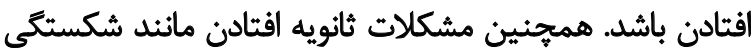

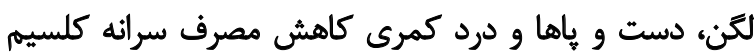

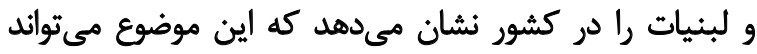

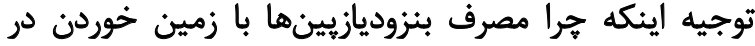

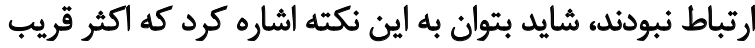

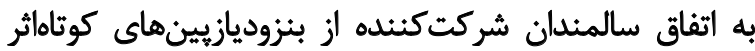

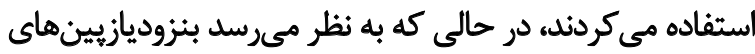

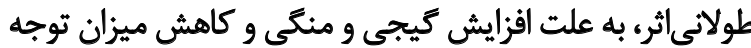

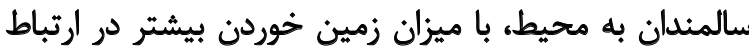

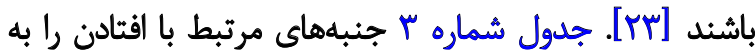

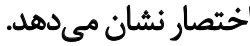

خانه علاوه بر اين كه مأمن آرامش است، مى بـواند خطرات

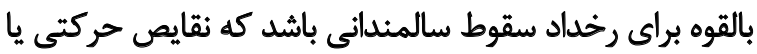

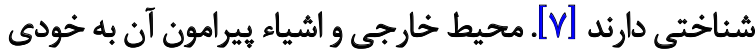

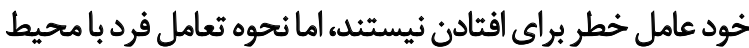

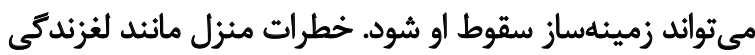

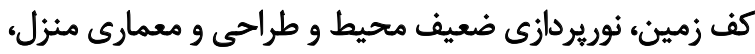

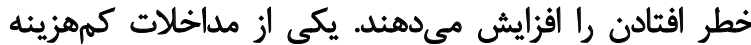

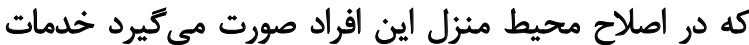

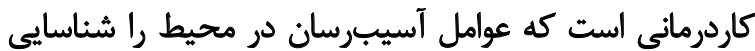

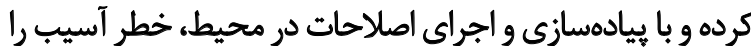

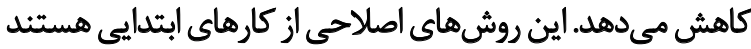

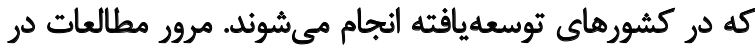

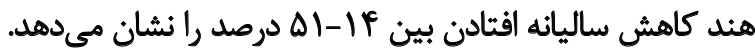

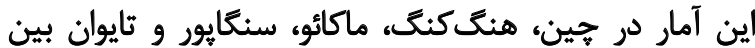

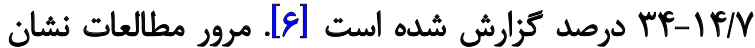

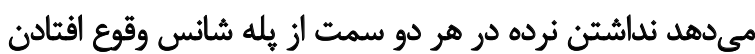

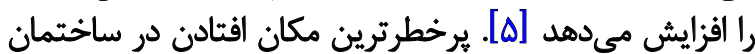

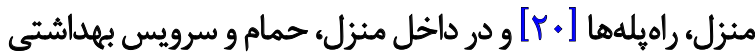

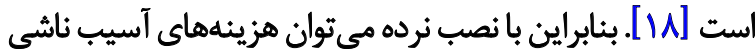

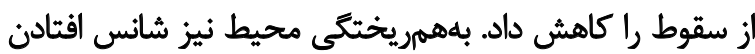

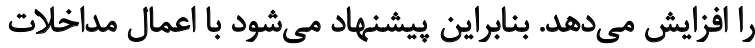

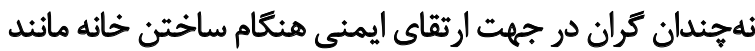

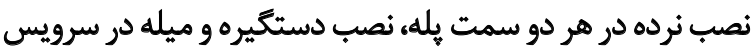

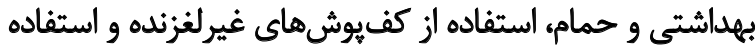

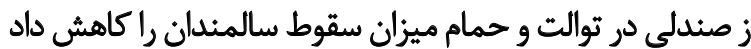

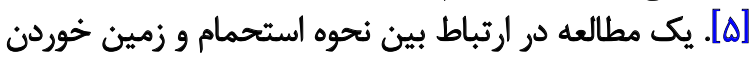

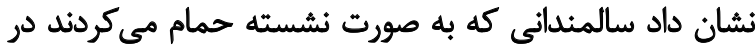

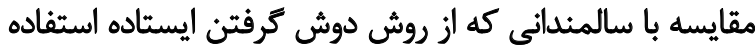

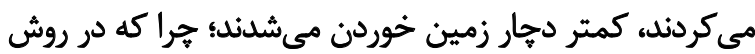

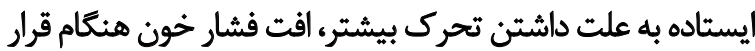

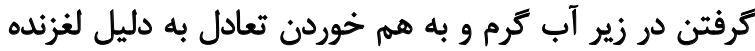

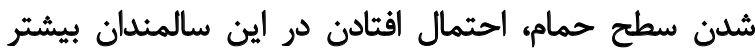

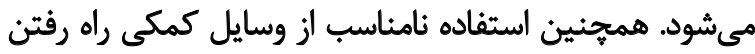

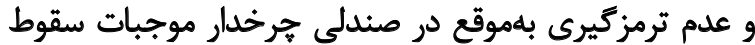

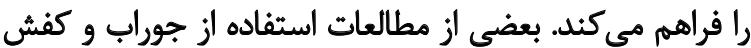

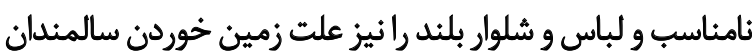


سلامت تغذيه را به جالش بكشائد و خطرات ناشى از سقوط را را

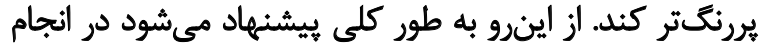

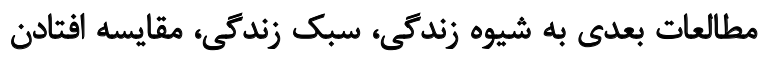

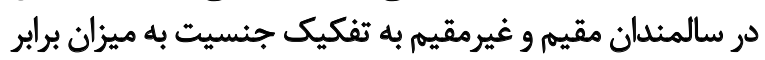

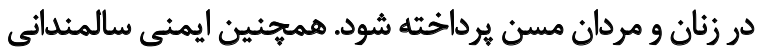

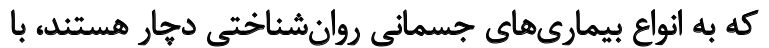

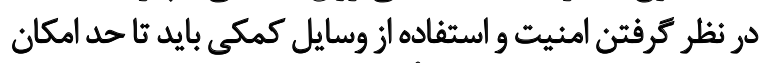

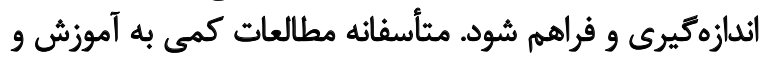

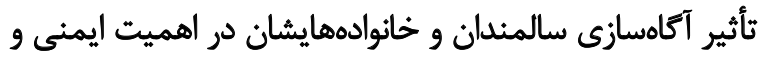

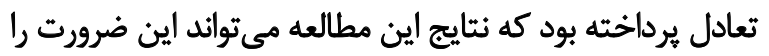
نمايان سازد.

مالاحظات اخلاقي بيروى أز الصول اخلاق بثروهش كليهى اصول اخلاقى در اين مقاله رعايت شده است.

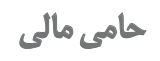

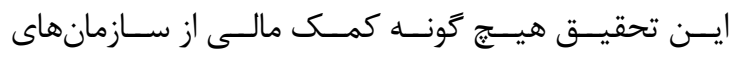

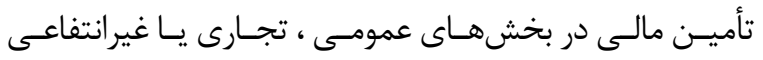

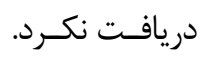

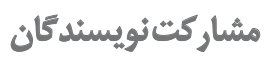

اعتبـار سـنجى، مفهومســازى، مديريـت برونده؛: ميناســادات

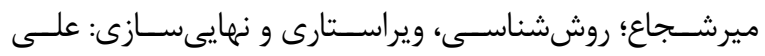

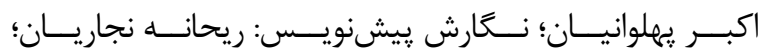

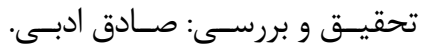

$$
\text { تمارض مناقع }
$$

بنابر اظهار نويسندكان، اين مقاله تعارض منافع ندارد.

$$
\text { تشكر و قدردافي }
$$

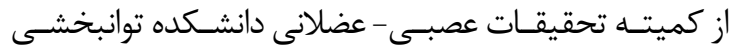

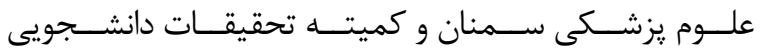

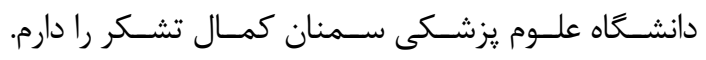




\section{References}

[1] Shaw BH, Borrel D, Sabbaghan K, Kum C, Yang Y, Robinovitch $\mathrm{SN}$, et al. Relationships between orthostatic hypotension, frailty, falling and mortality in elderly care home residents. BMC Geriatrics. 2019; 19(1):80. [DOI:10.1186/s12877-019-1082-6] [PMID] [PMCID]

[2] Marashi T, Ghadiri Sh, Ramezankhani A, Khodkarim S. [Study of fall and some of its related individual factors among the elderly attending to health centers affiliated to health network of Rey city in 2017 (Persian)]. Journal of Health in the Field. 2018; 6(2):49-57. https://journals.sbmu.ac.ir/en-jhf/article/view/23244

[3] Jafari M, Shamshiri M. [Reviewing the static and dynamic balance in predicting the risk of falls in elderly people in Tehran (Persian)]. Nursing and Midwifery Journal. 2015; 12(11):1045-53. http:// unmf.umsu.ac.ir/article-1-1995-en.html

[4] Kalula SZ, Ferreira M, Swingler GH, Badri M. Risk factors for falls in older adults in a South African Urban Community. BMC Geriatrics. 2016; 16:51. [DOI:10.1186/s12877-016-0212-7] [PMID] [PMCID]

[5] Iranfar M. [Physical hazards of residences and elderly fall (Persian)]. Journal of Safety Promotion and Injury Prevention. 2017; 5(4):237-42. [DOI:10.22037/meipm.v5i4.20720]

[6] Williams JS, Kowal P, Hestekin H, O’Driscoll T, Peltzer K, Yawson A, et al. Prevalence, risk factors and disability associated with fall-related injury in older adults in low-and middle-incomecountries: Results from the WHO Study on global AGEing and adult health (SAGE). BMC Medicine. 2015; 13:147. [DOI:10.1186/ s12916-015-0390-8] [PMID] [PMCID]

[7] Dhargave P, Sendhilkumar R. Prevalence of risk factors for falls among elderly people living in long-term care homes. Journal of Clinical Gerontology and Geriatrics. 2016; 7(3):99-103. [DOI:10.1016/j.jcgg.2016.03.004]

[8] Levinger P, Wallman S, Hill K. Balance dysfunction and falls in people with lower limb arthritis: Factors contributing to risk and effectiveness of exercise interventions. European Review of Aging and Physical Activity. 2012; 9:17-25. [DOI:10.1007/s11556011-0086-9]

[9] Taheri Tanjani P, Ainy E, Akbarpour S, Soori H. [Study of characteristics of falls among Iranian elders (Persian)]. Journal of Safety Promotion and Injury Prevention. 2015; 2(4):313-20. [DOI:10.22037/meipm.v2i4.8398]

[10] Grundstrom AC, Guse CE, Layde PM. Risk factors for falls and fall-related injuries in adults 85 years of age and older. Archives of Gerontology and Geriatrics. 2012; 54(3):421-8. [DOI:10.1016/j. archger.2011.06.008] [PMID] [PMCID]

[11] Nabavi SH, Hatami ST, Norouzi F, Gerivani Z, Hatami SE, Monadi Ziarat $\mathrm{H}$, et al. [Prevalence of fall and its related factors among older people in Bojnurd in 2015 (Persian)]. Salmand: Iranian Journal of Ageing. 2016; 11(3):466-73. [DOI:10.21859/ sija-1103466]

[12] Ghanbary A, Salehi Dehno N, Moslemi Haghighi F, Torabi $\mathrm{M}$. [The prevalence and correlates of falling down in the older adults over 55 years in Shiraz (Persian)]. Salmand: Iranian Journal of Ageing. 2013; 8(1):64-70. http://salmandj.uswr.ac.ir/article1-548-en.html
[13] Deshpande N, Metter EJ, Lauretani F, Bandinelli S, Guralnik J, Ferrucci L. Activity restriction induced by fear of falling and objective and subjective measures of physical function: A prospective cohort study. Journal of the American Geriatrics Society. 2008; 56(4):615-20. [DOI:10.1111/j.1532-5415.2007.01639.x] [PMID] [PMCID]

[14] Mohamed Hassan Saleh N, Shafik Ibrahim H, Abd El-Aziz Mohamed HN, El-Gilany AH. Predictors of fear of falling among community dwelling older adults in Mansoura city, Egypt. International Journal of Nursing Didactics. 2018; 8(08):63-71. [DOI:10.15520/ijnd.v8i08.2327]

[15] Ghodsi SM, Sayyar Roudsari B, Abdollahi M, Shadman M. Fallrelated injuries in the elderly in Tehran. Injury. 2003; 34(11):80914. [DOI:10.1016/S0020-1383(02)00376-5] [PMID]

[16] Akbari Kamrani AA, Azadi F, Foroughan M, Siadat S, Kaldi AR [Characteristics of falls among institutionalized elderly people (Persian)]. Salmand: Iranian Journal of Ageing. 2007; 1(2):101-5. http://salmandj.uswr.ac.ir/article-1-19-en.html

[17] Salarvand Sh, Birjandi M, Shamshiri M. [Assessing prevalence of fallings and their relation with chronic conditions for older people living in Khoramabad, Iran (Persian)]. Quarterly of the Horizon of Medical Sciences. 2008; 13(4):59-65. http://hms.gmu.ac.ir/ article-1-301-en.html

[18] Salarvand Sh, Birjandi M. [Factors related to falling down in older adults (Persian)]. Iran Journal of Nursing. 2009; 22(61):51-60 http://ijn.iums.ac.ir/article-1-737-en.html

[19] Esmaieli R, Abed Saeedi Zh, Esmaili M, Kaveh J. [A review study on effective factors in prevention of falling and osteoporosis fracture in elderly people (Persian)]. Scientific Journal of Rehabilitation Medicine. 2013; 1(4):47-56. http://medrehab.sbmu. ac.ir/article_1100102_en.html

[20] Jafarian Amiri S, Zabihi A, Aziznejad Roshan P, Hosseini S, Bijani A. [Fall at home and its related factors among the elderly in Babol city Iran (Persian)]. Journal of Babol University of Medical Sciences. 2013; 15(5):95-101. http://jbums.org/article1-4527-en.html

[21] Golmakani E, Usefi MR, Tabatabaeichehr M, Ghanei Zare F, Moayyed L, Hasanzadeh E, et al. [Fall in elderly : A literature review (Persian)]. Journal of North Khorasan University of Medical Sciences. 2014; 5(5):1159-63. [DOI:10.29252/ jnkums.5.5.S5.1159]

[22] Sayyah M, Khosravi GR, Bigdeli M. Frequency of fall-related injuries of female patients referred to the trauma center in the city of Kashan from years 2005 to 2008. Chinese Journal of Traumatology. 2013; 16(1):46-50. [PMID]

[23] Ghassemi S, Najafi B, Memari AR, Nazari N, Bakhtiari F, Alizadeh Khoei M, et al. [Elderly falls risk factors: A prospective longitudinal study in Kahrizak charity foundation (Persian)]. Iranian Journal of Diabetes and Metabolism. 2014; 13(6):447-54. http:// ijdld.tums.ac.ir/article-1-5262-en.htm

[24] Abdiani M, Abdiani E, Abdiani P, Hemmati M, Gholipour A Mokarm A. Investigating the fear of falling in the elderly in Charam (Persian)]. Paper presented at: $3^{\text {rd }}$ International Conference on Sport Sciences. December 2016; Tehran, Iran. https:// www.sid.ir $/ \mathrm{Fa} /$ Seminar $/$ ViewPaper.aspx?ID=29754 
[25] Sharifi F, Fakhrzadeh H, Memari AR, Najafi B, Nazari N, Alizadeh Khoee M, et al. Predicting risk of the fall among aged adult residents of a nursing home. Archives of Gerontology and Geriatrics. 2015; 61(2):124-30. [DOI:10.1016/j.archger.2015.06.014] [PMID]

[26] Davoodi F, Etemad K, Taheri Tanjani P, Khodakarim S. [The relationship between depression and cognitive impairment with falls leading to fractures in elderly (Persian)]. Journal of Safety Promotion and Injury Prevention. 2016; 4(2):75-82. [DOI:10.22037/ meipm.v4i2.14129]

[27] Hosseini SR, Ahmadi Ahangar A, Ghanbari N, Bijani A. [Prevalence of falls and its association with serum vitamin $\mathrm{D}$ levels in the elderly population of Amirkola city (Persian-English)]. Journal of Babol University of Medical Sciences. 2016; 18(8):20-8. [DOI:10.22088/jbums.18.8.20]

[28] Jafari Z, Mehrdad N, Sharifi F, Haghani H, Nikpour S. [Fall elated factors in the type 2 diabetes older adults: A case-control study on older adults referring to the hospital (Persian)]. Iranian Journal of Diabetes and Metabolism. 2016; 15(4):258-66. http:// ijdld.tums.ac.ir/article-1-5559-en.html

[29] Abbasi M, Daniali SSh, Hazrati M. [Lifestyle of fallen elderly patients referred to Isfahan hospitals (Persian)]. Iran Journal of Nursing. 2017; 30(107):20-31. [DOI:10.29252/ijn.30.107.20]

[30] Shirini AR, Arsham S, Yaali R. [The relationship between fear of falling, anxiety, functional mobility and balance in nursinghome older adults (Persian)]. Motor Behavior. 2017; 9(29):135-48. [DOI:10.22089/MBJ.2017.3227.1394]

[31] Mortazavi H, Tabatabaeichehr M, Taherpour M, Masoumi M. Relationship between home safety and prevalence of falls and fear of falling among elderly people: A cross-sectional study. Materia Socio Medica. 2018; 30(2):103-7. [DOI:10.5455/ msm.2018.30.103-107] [PMID] [PMCID]

[32] Taheri-Kharameh Z, Poorolajal J, Bashirian S, Heydari Moghadam R, Parham M, Barati M, et al. Risk factors for falls in Iranian older adults: A case-control study. International Journal of Injury Control and Safety Promotion. 2019; 26(4):354-9. [DOI:10.1080 /17457300.2019.1615958] [PMID]

[33] Na'emani F, Esmaiil Zali M, Sohrabi Z, Fayaz-Bakhsh A. [Prevalence of risk factors for falls among the elderly receiving care at home (Persian)]. Salmand: Iranian Journal of Ageing. 2019; 13(5):638-51. [DOI:10.32598/SIJA.13.Special-Issue.638]

[34] Bagheri Ruchi A, Mohammadi F, Mafi M, Motalebi SA. [Prevalence and external factors of home falls among the elderly in Qazvin (Persian)]. Journal of Inflammatory Diseases. 2019; 23(5):44051. [DOI:10.32598/JQUMS.23.5.440]

[35] Salavati M, Baharlouei H, Akhbari B. [The relationship between fear of falling and level of education and living style in the elderly (Persian)]. Research in Rehabilitation Sciences. 2015; 11(4):25762. http://jrrs.mui.ac.ir/index.php/jrrs/article/view/2064

[36] Coutinho ESF, Fletcher A, Bloch KV, Rodrigues LC. Risk factors for falls with severe fracture in elderly people living in a middle-income country: A case control study. BMC Geriatrics. 2008; 8:21. [DOI:10.1186/1471-2318-8-21] [PMID] [PMCID]

[37] Hasankhani H, Malek M, Asghari Jafarabadi M, Darvishpur Kakhki A. [Investigating the relationship between fear of falling and quality of life in community dwelling elders (Persian)]. Journal of Geriatric Nursing. 2014; 1(1):9-19. http://jgn.medilam. ac.ir/article-1-53-en.html

[38] Pua YH, Ong PH, Clark RA, Matcher DB, Lim ECW. Falls efficacy, postural balance, and risk for falls in older adults with fallsrelated emergency department visits: Prospective cohort study. BMC Geriatrics. 2017; 17(1):291. [DOI:10.1186/s12877-0170682-2] [PMID] [PMCID]

[39] Paul S. Falls: Prevention and management. In: Nair B, editor. Geriatric Medicine. Singapore: Springer; 2018. p. 109-119. [DOI:10.1007/978-981-10-3253-0_8] 